ESAIM: M2AN 50 (2016) 699-725

DOI: $10.1051 / \mathrm{m} 2 \mathrm{an} / 2015059$
ESAIM: Mathematical Modelling and Numerical Analysis

www.esaim-m2an.org

\title{
$h p$-VERSION DISCONTINUOUS GALERKIN METHODS FOR ADVECTION-DIFFUSION-REACTION PROBLEMS ON POLYTOPIC MESHES
}

\author{
Andrea Cangiani ${ }^{1}$, ZhaOnan Dong ${ }^{1}$, \\ Emmanuil H. Georgoulis ${ }^{2}$ and Paul Houston ${ }^{3}$
}

\begin{abstract}
We consider the $h p$-version interior penalty discontinuous Galerkin finite element method (DGFEM) for the numerical approximation of the advection-diffusion-reaction equation on general computational meshes consisting of polygonal/polyhedral (polytopic) elements. In particular, new $h p$-version a priori error bounds are derived based on a specific choice of the interior penalty parameter which allows for edge/face-degeneration. The proposed method employs elemental polynomial bases of total degree $p\left(\mathcal{P}_{p}\right.$-basis $)$ defined in the physical coordinate system, without requiring the mapping from a given reference or canonical frame. Numerical experiments highlighting the performance of the proposed DGFEM are presented. In particular, we study the competitiveness of the $p$-version DGFEM employing a $\mathcal{P}_{p}$-basis on both polytopic and tensor-product elements with a (standard) DGFEM employing a (mapped) $\mathcal{Q}_{p}$-basis. Moreover, a computational example is also presented which demonstrates the performance of the proposed $h p$-version DGFEM on general agglomerated meshes.
\end{abstract}

Mathematics Subject Classification. 65N30, 65N50, 65N55.

Received April 14, 2015. Revised August 10, 2015.

Published online May 23, 2016.

\section{INTRODUCTION}

Discontinuous Galerkin methods have enjoyed considerable success, especially during the last 15 years, and are now considered a standard variational framework for the numerical solution of many classes of problems involving partial differential equations (PDEs). The origins of the discontinuous Galerkin finite element method (DGFEM, for short) can be traced back to the early 1970s for the numerical solution of first-order hyperbolic

Keywords and phrases. Discontinuous Galerkin; polygonal elements; polyhedral elements; $h p$-finite element methods; inverse estimates; $\mathcal{P}$-basis; PDEs with nonnegative characteristic form.

1 Department of Mathematics, University of Leicester, Leicester LE1 7RH, UK. Andrea.Cangiani@le.ac.uk; zd14@le.ac.uk

2 Department of Mathematics, University of Leicester, Leicester LE1 7RH, UK \& School of Applied Mathematical and Physical

Sciences, National Technical University of Athens, 15780 Athens, Greece. Emmanuil.Georgoulis@le.ac.uk

3 School of Mathematical Sciences, University of Nottingham, Nottingham, NG7 2RD, UK. Paul.Houston@nottingham.ac.uk 
problems [45] and for the weak imposition of inhomogeneous boundary conditions for elliptic problems [42]. The use of discontinuous/nonconforming approximation spaces in the context of finite element methods also first appeared around the same time [10,13]; for reviews of some of the main developments in the subject, we refer to the monographs $[25,28]$ and the articles $[8,24]$.

The interest in DGFEMs can be attributed to a number of factors: classical DGFEMs, such as interior penalty methods, have typically minimal communication, in the sense that only direct face-element neighbours are coupled through the exploitation of appropriate numerical fluxes; this has important advantages for imposing boundary conditions and also for parallel efficiency. Additionally, DGFEMs can incorporate a wealth of numerical fluxes into their formulation, leading to stable discretizations in the context of multi-scale problems. Moreover, meshes containing hanging-nodes and elemental polynomial bases consisting of locally variable polynomial degrees are also admissible, owing to the lack of pointwise continuity requirements across the mesh-skeleton. Also, powerful solvers are now available for the resulting linear systems; indeed, both domain decomposition preconditioners, see, for example, $[1-3,5,29,39]$, and the references cited therein, as well as multigrid solvers, cf. $[6,7,17,18]$, have been developed.

More recently, DGFEMs on meshes containing extremely general element shapes, such as general polygons in two dimensions and polyhedra in three dimensions, have been proposed $[4,14-16,21,28,33,40,50]$. Such meshes can naturally be combined with DGFEMs due to their element-wise discontinuous approximation. To support such a variety of element shapes, without any detrimental affect on the local approximation properties of the underlying DGFEM, polynomial spaces defined in the physical frame, rather than mapped polynomials from a reference element, are typically employed. In our recent work [21], an $h p$-version DGFEM of interior penalty (IP) type for linear elliptic problems on meshes consisting of $d$-dimensional polytopic elements (i.e., polygons for $d=2$ and polyhedra for $d=3$ ) was proposed and analysed. A key aspect of the method proposed in [21], is that the scheme remains well-defined and practical in the presence of arbitrarily small/degenerate $(d-k)$-dimensional element facets, $k=1, \ldots, d-1$, where $d$ denotes the spatial dimension. A by-product of the approach developed in [21] was the proposal of physical frame $\mathcal{P}_{p}$-type bases, i.e., local polynomial spaces of total degree $p$, for the case when the underlying polytopes are simply quadrilateral/hexahedral, compared to the standard approach of employing a mapped tensor-product $\mathcal{Q}_{p}$ polynomial basis, i.e., tensor-product polynomials of degree $p$ in each spatial variable. Indeed, it was demonstrated numerically that the DGFEM employing a $\mathcal{P}_{p}$-type basis achieves a faster rate of convergence, with respect to the number of degrees of freedom present in the underlying finite element space, as the polynomial degree $p$ increases, for a given fixed mesh, than the respective DGFEM employing a (mapped) $\mathcal{Q}_{p}$ basis on tensor-product elements.

In this work, we extend the results of [21] to cover $h p$-version IP DGFEMs for a general class of linear advection-diffusion-reaction PDE problems, often referred to as equations with nonnegative characteristic form. DGFEMs for this class of problems on quadrilateral meshes were first proposed and analysed in [36]. Here, we derive a priori bounds for the $h p$-version IP DGFEM for this class of PDE problems. Due to the lack of $h p$-approximation results for the local $L_{2}$-projection operator on polytopic elements, it is not possible to directly generalise the analysis from [36] to meshes consisting of such elements. To address this issue, we prove an inf-sup condition for the underlying DGFEM, with respect to a stronger streamline-diffusion type norm, for simple advection coefficients, thereby extending respective results from $[9,19,20,37]$ to the current setting. This naturally leads to a priori bounds for the $h p$-version DGFEM for this general class of linear PDE problems on very general polytopic meshes with possibly arbitrarily small/degenerate $(d-k)$-dimensional element facets, $k=1, \ldots, d-1$.

This work is structured as follows. In Section 2, we introduce the model problem and define the set of admissible subdivisions of the computational domain. In Section 3 we formulate the IP DGFEM; Section 4 presents relevant $h p$-approximation results. The stability and a priori analysis of the proposed method is then undertaken in Section 5. The practical performance of the IP DGFEM is studied in Section 6 through a series of numerical examples. Finally, in Section 7 we summarize the work presented in this paper and draw some conclusions. 


\section{Preliminaries}

For a Lipschitz domain $\omega \subset \mathbb{R}^{d}, d \geq 1$, we denote by $H^{s}(\omega)$ the Hilbertian Sobolev space of index $s \geq 0$ of real-valued functions defined on $\omega$, endowed with the seminorm $|\cdot|_{H^{s}(\omega)}$ and norm $\|\cdot\|_{H^{s}(\omega)}$. Furthermore, we let $L_{p}(\omega), p \in[1, \infty]$, be the standard Lebesgue space on $\omega$, equipped with the norm $\|\cdot\|_{L_{p}(\omega)}$. Finally, $|\omega|$ denotes the $d$-dimensional Hausdorff measure of $\omega$.

\subsection{Model problem}

Let $\Omega$ be a bounded open polyhedral domain in $\mathbb{R}^{d}, d=2,3$, and let $\Gamma$ signify the union of its $(d-1)$ dimensional open faces. We consider the advection-diffusion-reaction equation

$$
\mathcal{L} u \equiv-\nabla \cdot(a \nabla u)+\mathbf{b} \cdot \nabla u+c u=f, \quad \text { in } \Omega,
$$

where $c \in L_{\infty}(\Omega), f \in L_{2}(\Omega)$, and $\mathbf{b}:=\left(b_{1}, b_{2}, \ldots, b_{d}\right)^{\top} \in\left[W_{\infty}^{1}(\Omega)\right]^{d}$. Here, $a=\left\{a_{i j}\right\}_{i, j=1}^{d}$ is a symmetric positive semidefinite tensor whose entries $a_{i j}$ are bounded, piecewise continuous, real-valued functions defined on $\bar{\Omega}$, with

$$
\xi^{\top} a(x) \xi \geq 0 \quad \forall \xi \in \mathbb{R}^{d}, \quad \text { a.e. } \quad x \in \bar{\Omega} .
$$

Under the above hypothesis, (2.1) is termed a partial differential equation with nonnegative characteristic form.

We denote by $\mathbf{n}(x)=\left\{n_{i}(x)\right\}_{i=1}^{d}$ the unit outward normal vector to $\Gamma$ at $x \in \Gamma$ and introduce the Fichera function $\mathbf{b} \cdot \mathbf{n}$ to define

$$
\begin{gathered}
\Gamma_{0}=\left\{x \in \Gamma: \mathbf{n}(x)^{\top} a(x) \mathbf{n}(x)>0\right\}, \\
\Gamma_{-}=\left\{x \in \Gamma \backslash \Gamma_{0}: \mathbf{b}(x) \cdot \mathbf{n}(x)<0\right\}, \quad \Gamma_{+}=\left\{x \in \Gamma \backslash \Gamma_{0}: \mathbf{b}(x) \cdot \mathbf{n}(x) \geq 0\right\} .
\end{gathered}
$$

The sets $\Gamma_{-}$and $\Gamma_{+}$are referred to as the inflow and outflow boundary, respectively. Note that $\Gamma=\Gamma_{0} \cup \Gamma_{-} \cup \Gamma_{+}$. If $\Gamma_{0}$ is nonempty, we subdivide it into two disjoint subsets $\Gamma_{\mathrm{D}}$ and $\Gamma_{\mathrm{N}}$ whose union is $\Gamma_{0}$, with $\Gamma_{\mathrm{D}}$ nonempty and relatively open in $\Gamma$, on which we consider the boundary conditions for (2.1):

$$
u=g_{\mathrm{D}} \quad \text { on } \quad \Gamma_{\mathrm{D}} \cup \Gamma_{-}, \quad \mathbf{n} \cdot(a \nabla u)=g_{\mathrm{N}} \quad \text { on } \quad \Gamma_{\mathrm{N}},
$$

and also adopt the hypothesis that $\mathbf{b} \cdot \mathbf{n} \geq 0$ on $\Gamma_{\mathrm{N}}$, whenever $\Gamma_{\mathrm{N}}$ is nonempty. Additionally, we assume that the following positivity hypothesis holds: there exists a positive constant $\gamma_{0}$ such that

$$
c_{0}(x)^{2}:=c(x)-\frac{1}{2} \nabla \cdot \mathbf{b}(x) \geq \gamma_{0} \quad \text { a.e. } x \in \Omega .
$$

The well-posedness of the boundary value problem (2.1), (2.4) has been studied in [35].

\subsection{Finite element spaces}

Let $\mathcal{T}$ be a subdivision of the computational domain $\Omega$ into disjoint open polygonal $(d=2)$ or polyhedral $(d=3)$ elements $\kappa$ such that $\bar{\Omega}=\cup_{\kappa \in \mathcal{T}} \bar{\kappa}$ and denote by $h_{\kappa}$ the diameter of $\kappa \in \mathcal{T}$; i.e., $h_{\kappa}:=\operatorname{diam}(\kappa)$. In the absence of hanging nodes/edges, we define the interfaces of the mesh $\mathcal{T}$ to be the set of $(d-1)$-dimensional facets of the elements $\kappa \in \mathcal{T}$. To facilitate the presence of hanging nodes/edges, which are permitted in $\mathcal{T}$, the interfaces of $\mathcal{T}$ are defined to be the intersection of the $(d-1)$-dimensional facets of neighbouring elements. In the case when $d=2$, the interfaces of a given element $\kappa \in \mathcal{T}$ will always consist of line segments $((d-1)$ dimensional simplices). For $d=3$, we assume that each interface of an element $\kappa \in \mathcal{T}$ may be subdivided into a set of co-planar triangles. With this in mind we use the terminology 'face' to refer to a $(d-1)$-dimensional simplex (line segment or triangle for $d=2$ or 3 , respectively), which forms part of the boundary (interface) of an element $\kappa \in \mathcal{T}$. For $d=2$, the face and interface of an element $\kappa \in \mathcal{T}$ necessarily coincide with each 
other, while in three-dimensions this may no longer be the case, since the boundary of a general polyhedron may consist of planar polygons which are not triangular.

As in [21], we assume that a sub-triangulation into faces of each mesh interface is given if $d=3$, and denote by $\mathcal{E}$ the union of all open mesh interfaces if $d=2$ and the union of all open triangles belonging to the subtriangulation of all mesh interfaces if $d=3$. In this way, $\mathcal{E}$ is always defined as a set of $(d-1)$-dimensional simplices. Further, we write $\mathcal{E}_{\text {int }}$ to denote the union of all open $(d-1)$-dimensional element faces $F \subset \mathcal{E}$ that are contained in $\Omega$, and let $\Gamma_{\text {int }}:=\left\{x \in \Omega: x \in F, F \in \mathcal{E}_{\text {int }}\right\}$. Further assumptions on the class of admissible meshes will be outlined later on in Section 4 .

Given $\kappa \in \mathcal{T}$, we write $p_{\kappa}$ to denote the (positive) polynomial degree of the element $\kappa$, and collect the $p_{\kappa}$ in the vector $\mathbf{p}:=\left(p_{\kappa}: \kappa \in \mathcal{T}\right)$. We then define the finite element space $S_{\mathcal{T}}^{\mathbf{p}}$ with respect to $\mathcal{T}$ and $\mathbf{p}$ by

$$
S_{\mathcal{T}}^{\mathbf{p}}:=\left\{u \in L_{2}(\Omega):\left.u\right|_{\kappa} \in \mathcal{P}_{p_{\kappa}}(\kappa), \kappa \in \mathcal{T}\right\},
$$

where $\mathcal{P}_{p_{\kappa}}(\kappa)$ denotes the space of polynomials of total degree $p_{\kappa}$ on $\kappa$. As in [21], we point out that the local elemental polynomial spaces employed within the definition of $S_{\mathcal{T}}^{\mathrm{p}}$ are defined in the physical coordinate system, without the need to map from a given reference or canonical frame. We define the broken Sobolev space $H^{\mathbf{s}}(\Omega, \mathcal{T})$ with respect to the subdivision $\mathcal{T}$ up to composite order $\mathbf{s}$ as follows

$$
H^{\mathbf{s}}(\Omega, \mathcal{T})=\left\{u \in L_{2}(\Omega):\left.u\right|_{\kappa} \in H^{s_{\kappa}}(\kappa) \quad \forall \kappa \in \mathcal{T}\right\} .
$$

For $u \in H^{1}(\Omega, \mathcal{T})$, we define the broken gradient $\nabla_{h} u$ by $\left.\left(\nabla_{h} u\right)\right|_{\kappa}=\nabla\left(\left.u\right|_{\kappa}\right), \kappa \in \mathcal{T}$, which will be used to construct the forthcoming DGFEM.

\subsection{Trace operators}

For any element $\kappa \in \mathcal{T}$, we denote by $\partial \kappa$ the union of $(d-1)$-dimensional open faces of $\kappa$. Then, the inflow and outflow parts of $\partial \kappa$ are defined as follows

$$
\partial_{-} \kappa=\left\{x \in \partial \kappa, \quad \mathbf{b}(x) \cdot \mathbf{n}_{\kappa}(x)<0\right\}, \quad \partial_{+} \kappa=\left\{x \in \partial \kappa, \quad \mathbf{b}(x) \cdot \mathbf{n}_{\kappa}(x) \geq 0\right\},
$$

respectively, where $\mathbf{n}_{\kappa}(x)$ denotes the unit outward normal vector to $\partial \kappa$ at $x \in \partial \kappa$. Given $\kappa \in \mathcal{T}$, the trace of a function $v \in H^{1}(\Omega, \mathcal{T})$ on $\partial_{-} \kappa$, relative to $\kappa$, is denoted by $v_{\kappa}^{+}$. Further, if $\partial_{-} \kappa \backslash \Gamma$ is nonempty, then for $x \in \partial_{-} \kappa \backslash \Gamma$ there exits a unique $\kappa^{\prime} \in \mathcal{T}$ such that $x \in \partial_{+} \kappa^{\prime}$; with this notation, we denote by $v_{\kappa}^{-}$the trace of $\left.v\right|_{\kappa^{\prime}}$ on $\partial_{-} \kappa \backslash \Gamma$. Hence the upwind jump of the (scalar-valued) function $v$ across a face $F \subset \partial_{-} \kappa \backslash \Gamma$ is denoted by

$$
\lfloor v\rfloor:=v_{\kappa}^{+}-v_{\kappa}^{-} .
$$

Next, we introduce some additional trace operators. Let $\kappa_{i}$ and $\kappa_{j}$ be two adjacent elements of $\mathcal{T}$ and let $x$ be an arbitrary point on the interior face $F \subset \Gamma_{\text {int }}$ given by $F=\partial \kappa_{i} \cap \partial \kappa_{j}$. We write $\mathbf{n}_{i}$ and $\mathbf{n}_{j}$ to denote the outward unit normal vectors on $F$, relative to $\partial \kappa_{i}$ and $\partial \kappa_{j}$, respectively. Furthermore, let $v$ and $\mathbf{q}$ be scalarand vector-valued functions, which are smooth inside each element $\kappa_{i}$ and $\kappa_{j}$. By $\left(v_{i}, \mathbf{q}_{i}\right)$ and $\left(v_{j}, \mathbf{q}_{j}\right)$, we denote the traces of $(v, \mathbf{q})$ on $F$ taken from within the interior of $\kappa_{i}$ and $\kappa_{j}$, respectively. The averages of $v$ and $\mathbf{q}$ at $x \in F$ are given by

$$
\left\{\{v\}:=\frac{1}{2}\left(v_{i}+v_{j}\right), \quad\{\mathbf{q}\}\right\}:=\frac{1}{2}\left(\mathbf{q}_{i}+\mathbf{q}_{j}\right),
$$

respectively. Similarly, the jump of $v$ and $\mathbf{q}$ at $x \in F \subset \Gamma_{\text {int }}$ are given by

$$
\llbracket v \rrbracket:=v_{i} \mathbf{n}_{i}+v_{j} \mathbf{n}_{j}, \quad \llbracket \mathbf{q} \rrbracket:=\mathbf{q}_{i} \cdot \mathbf{n}_{i}+\mathbf{q}_{j} \cdot \mathbf{n}_{j},
$$

respectively. On a boundary face $F \subset \Gamma$, such that $F \subset \partial \kappa_{i}, \kappa_{i} \in \mathcal{T}$, we set

$$
\{\{v\}\}=v_{i}, \quad\left\{\{\mathbf{q}\}=\mathbf{q}_{i}, \quad \llbracket v \rrbracket=v_{i} \mathbf{n}_{i} \quad \llbracket \mathbf{q} \rrbracket=\mathbf{q}_{i} \cdot \mathbf{n}_{i},\right.
$$

with $\mathbf{n}_{i}$ denoting the unit outward normal vector on the boundary $\Gamma$.

Remark 2.1. The jump operator $\llbracket \cdot \rrbracket$ is independent of face orientation, while the sign of the upwind jump operator $\lfloor\cdot\rfloor$ depends on the direction of the flow. 


\section{INTERIOR PENALTy DisCONTINUOUS GALERKIN METHOD}

In this section, we introduce the $h p$-version DGFEM discretization of the model problem (2.1), (2.4). For simplicity of presentation, we suppose that the entries of the diffusion tensor $a$ are constant on each element $\kappa \in \mathcal{T}$, i.e.,

$$
a \in\left[S_{\mathcal{T}}^{\mathbf{0}}\right]_{\mathrm{sym}}^{d \times d} .
$$

Our results can easily be extended to the case of general $a \in L_{\infty}(\Omega)_{\text {sym }}^{d \times d}$ based on employing the modified DGFEM proposed in [32]. In the following, $\sqrt{a}$ denotes the (positive semidefinite) square-root of the symmetric tensor $a$; further, $\bar{a}_{\kappa}:=\left.|\sqrt{a}|_{2}^{2}\right|_{\kappa}$, where $|\cdot|_{2}$ denotes the $l_{2}$-norm.

The IP DGFEM is given by: find $u_{h} \in S_{\mathcal{T}}^{\text {p }}$ such that

$$
B\left(u_{h}, v_{h}\right)=\ell\left(v_{h}\right)
$$

for all $v_{h} \in S_{\mathcal{T}}^{\mathbf{p}}$. Here, the bilinear form $B(\cdot, \cdot): S_{\mathcal{T}}^{\mathbf{p}} \times S_{\mathcal{T}}^{\mathbf{p}} \rightarrow \mathbb{R}$ is defined as the sum of two parts:

$$
B(u, v):=B_{\text {ar }}(u, v)+B_{\mathrm{d}}(u, v),
$$

where the bilinear form $B_{\mathrm{ar}}(\cdot, \cdot)$ accounts for the advection and reaction terms:

$$
\begin{aligned}
B_{\operatorname{ar}}(u, v):= & \sum_{\kappa \in \mathcal{T}} \int_{\kappa}(\mathbf{b} \cdot \nabla u+c u) v \mathrm{~d} x \\
& -\sum_{\kappa \in \mathcal{T}} \int_{\partial_{-} \backslash \Gamma}(\mathbf{b} \cdot \mathbf{n})\lfloor u\rfloor v^{+} \mathrm{d} s-\sum_{\kappa \in \mathcal{T}} \int_{\partial_{-} \kappa \cap\left(\Gamma_{\mathrm{D}} \cup \Gamma_{-}\right)}(\mathbf{b} \cdot \mathbf{n}) u^{+} v^{+} \mathrm{d} s .
\end{aligned}
$$

The bilinear form $B_{\mathrm{d}}(\cdot, \cdot)$ takes care of the diffusion term:

$$
\begin{aligned}
B_{\mathrm{d}}(u, v):= & \sum_{\kappa \in \mathcal{T}} \int_{\kappa} a \nabla u \cdot \nabla v \mathrm{~d} x+\int_{\Gamma_{\mathrm{int}} \cup \Gamma_{\mathrm{D}}} \sigma \llbracket u \rrbracket \cdot \llbracket v \rrbracket \mathrm{d} s \\
& -\int_{\Gamma_{\mathrm{int}} \cup \Gamma_{\mathrm{D}}}\left(\left\{\left\{a \nabla_{h} u\right\}\right\} \llbracket \llbracket v \rrbracket+\left\{\left\{a \nabla_{h} v\right\}\right\} \cdot \llbracket u \rrbracket\right) \mathrm{d} s .
\end{aligned}
$$

Furthermore, the linear functional $\ell: S_{\mathcal{T}}^{\mathrm{p}} \rightarrow \mathbb{R}$ is defined by

$$
\begin{aligned}
\ell(v):= & \sum_{\kappa \in \mathcal{T}} \int_{\kappa} f v \mathrm{~d} x-\sum_{\kappa \in \mathcal{T}} \int_{\partial_{-} \kappa \cap\left(\Gamma_{\mathrm{D}} \cup \Gamma_{-}\right)}(\mathbf{b} \cdot \mathbf{n}) g_{\mathrm{D}} v^{+} \mathrm{d} s \\
& -\int_{\Gamma_{\mathrm{D}}} g_{\mathrm{D}}\left(\left(a \nabla_{h} v\right) \cdot \mathbf{n}-\sigma v\right) \mathrm{d} s+\int_{\Gamma_{\mathrm{N}}} g_{\mathrm{N}} v \mathrm{~d} s .
\end{aligned}
$$

The nonnegative function $\sigma \in L_{\infty}\left(\Gamma_{\mathrm{int}} \cup \Gamma_{\mathrm{D}}\right)$ appearing in (3.4) and (3.5) is referred to as the discontinuitypenalization parameter; its precise definition, which depends on the diffusion tensor $a$ and the discretization parameters, will be given in Lemma 5.1.

\section{Approximation And inVerse estimates}

In this section, we revisit some polynomial approximation and inverse estimates in the context of general polytopic elements from [21]. Furthermore, we derive a new extension of a standard inverse estimate for polynomial functions. To this end, we introduce the following set of mesh assumptions. 
Assumption 4.1. The subdivision $\mathcal{T}$ is shape regular in the sense of [23], i.e., there exists a positive constant $C_{\text {shape }}$, independent of the mesh parameters, such that:

$$
\forall \kappa \in \mathcal{T}, \quad \frac{h_{\kappa}}{\rho_{\kappa}} \leq C_{\text {shape }},
$$

with $\rho_{\kappa}$ denoting the diameter of the largest ball contained in $\kappa$.

Assumption 4.2. There exists a positive constant $C_{F}$, independent of the mesh parameters, such that

$$
\max _{\kappa \in \mathcal{T}}\left(\operatorname{card}\left\{F \subset \Gamma \cup \Gamma_{\text {int }}: F \subset \partial \kappa\right\}\right) \leq C_{F} .
$$

Remark 4.3. We note that Assumption 4.2 naturally imposes the condition that the number of hanging nodes and the number of faces that each element $\kappa$ in the finite element mesh $\mathcal{T}$ possesses is uniformly bounded under mesh refinement.

As in [21], we require the existence of the following coverings of the mesh.

Definition 4.4. A (typically overlapping) covering $\mathcal{T}_{\sharp}=\{\mathcal{K}\}$ related to the polytopic mesh $\mathcal{T}$ is a set of shaperegular $d$-simplices $\mathcal{K}$, such that for each $\kappa \in \mathcal{T}$, there exists a $\mathcal{K} \in \mathcal{T}_{\sharp}$, with $\kappa \subset \mathcal{K}$. Given $\mathcal{T}_{\sharp}$, we denote by $\Omega_{\sharp}$ the covering domain given by $\Omega_{\sharp}:=\left(\cup_{\mathcal{K} \in \mathcal{T}_{\sharp}} \overline{\mathcal{K}}\right)^{\circ}$, where, for a closed set $D \subset \mathbb{R}^{d}, D^{\circ}$ denotes the interior of $D$.

Assumption 4.5. There exists a covering $\mathcal{T}_{\sharp}$ of $\mathcal{T}$ and a positive constant $\mathcal{O}_{\Omega}$, independent of the mesh parameters, such that the subdivision $\mathcal{T}$ satisfies

$$
\max _{\kappa \in \mathcal{T}} \mathcal{O}_{\kappa} \leq \mathcal{O}_{\Omega}
$$

where, for each $\kappa \in \mathcal{T}$,

$$
\mathcal{O}_{\kappa}:=\operatorname{card}\left\{\kappa^{\prime} \in \mathcal{T}: \kappa^{\prime} \cap \mathcal{K} \neq \emptyset, \mathcal{K} \in \mathcal{T}_{\sharp} \text { such that } \kappa \subset \mathcal{K}\right\} .
$$

As a consequence, we deduce that

$$
\operatorname{diam}(\mathcal{K}) \leq C_{\text {diam }} h_{\kappa},
$$

for each pair $\kappa \in \mathcal{T}, \mathcal{K} \in \mathcal{T}_{\sharp}$, with $\kappa \subset \mathcal{K}$, for a constant $C_{\text {diam }}>0$, uniformly with respect to the mesh size.

We note that Assumption 4.5 ensures that the amount of overlap present in the covering $\mathcal{T}_{\sharp}$ remains bounded as the computational mesh $\mathcal{T}$ is refined. The proceeding $h p$-approximation results and inverse estimates for polytopic elements are based on referring back to $d$-dimensional simplices, where standard results can be applied; see, for example, $[11,12,22,41]$. With this in mind, we introduce the following definition.

Definition 4.6. For each element $\kappa$ in the computational mesh $\mathcal{T}$, we define the family $\mathcal{F}_{b}^{\kappa}$ of all possible $d$-dimensional simplices contained in $\kappa$ and having at least one face in common with $\kappa$. The notation $\kappa_{b}^{F}$ will be used to indicate a simplex belonging to $\mathcal{F}_{b}^{\kappa}$ and sharing with $\kappa \in \mathcal{T}$ a given face $F$.

Functions defined on $\Omega$ can be extended to the covering domain $\Omega_{\sharp}$ based on employing the following extension operator, $c f .[47]$.

Theorem 4.7. Let $\Omega$ be a domain with a Lipschitz boundary. Then there exists a linear extension operator $\mathfrak{E}: H^{s}(\Omega) \rightarrow H^{s}\left(\mathbb{R}^{d}\right), s \in \mathbb{N}_{0}$, such that $\left.\mathfrak{E} v\right|_{\Omega}=v$ and

$$
\|\mathfrak{E} v\|_{H^{s}\left(\mathbb{R}^{d}\right)} \leq C\|v\|_{H^{s}(\Omega)},
$$

where $C$ is a positive constant depending only on $s$ and $\Omega$.

With the above notation, we now quote Lemma 4.2 from [21]. 
Lemma 4.8. Let $\kappa \in \mathcal{T}, F \subset \partial \kappa$ denote one of its faces, and $\mathcal{K} \in \mathcal{T}_{\sharp}$ denote the corresponding simplex such that $\kappa \subset \mathcal{K}$, cf. Definition 4.4. Suppose that $v \in L_{2}(\Omega)$ is such that $\left.\mathfrak{E} v\right|_{\mathcal{K}} \in H^{l_{\kappa}}(\mathcal{K})$, for some $l_{\kappa} \geq 0$. Then, given Assumption 4.5 is satisfied, there exists $\tilde{\Pi} v$, such that $\left.\tilde{\Pi} v\right|_{\kappa} \in \mathcal{P}_{p_{\kappa}}(\kappa)$, and the following bounds hold

$$
\|v-\tilde{\Pi} v\|_{H^{q}(\kappa)} \leq C \frac{h_{\kappa}^{s_{\kappa}-q}}{p_{\kappa}^{l_{\kappa}-q}}\|\mathfrak{E} v\|_{H^{l_{\kappa}(\mathcal{K})}}, \quad l_{\kappa} \geq 0,
$$

for $0 \leq q \leq l_{\kappa}$, and

$$
\|v-\tilde{\Pi} v\|_{L_{2}(F)} \leq C|F|^{1 / 2} \frac{h_{\kappa}^{s_{\kappa}-d / 2}}{p_{\kappa}^{l_{\kappa}-1 / 2}} C_{m}\left(p_{\kappa}, \kappa, F\right)^{1 / 2}\|\mathfrak{E} v\|_{H^{l_{\kappa}(\mathcal{K})}}, \quad l_{\kappa}>d / 2,
$$

where

$$
C_{m}\left(p_{\kappa}, \kappa, F\right)=\min \left\{\frac{h_{\kappa}^{d}}{\sup _{\kappa_{b}^{F} \subset \kappa}\left|\kappa_{b}^{F}\right|}, \frac{1}{p_{\kappa}^{1-d}}\right\} .
$$

Here, $s_{\kappa}=\min \left\{p_{\kappa}+1, l_{\kappa}\right\}$ and $C$ is a positive constant that depends on the shape-regularity of $\mathcal{K}$, but is independent of $v, h_{\kappa}$, and $p_{\kappa}$.

We now consider the derivation of $h p$-version inverse estimates, which are sharp with respect to $(d-k)$ dimensional, $k=1, \ldots, d-1$, element facet degeneration. To this end, we first recall the following definition, cf. $[21]$.

Definition 4.9. We let $\tilde{\mathcal{T}}$ denote the subset of elements $\kappa, \kappa \in \mathcal{T}$, such that each $\kappa \in \tilde{\mathcal{T}}$ can be covered by at most $m_{\mathcal{T}}$ shape-regular simplices $K_{i}, i=1, \ldots, m_{\mathcal{T}}$, such that

$$
\operatorname{dist}\left(\kappa, \partial K_{i}\right)>C_{a s} \operatorname{diam}\left(K_{i}\right) / p_{\kappa}^{2},
$$

and

$$
\left|K_{i}\right| \geq c_{a s}|\kappa|
$$

for all $i=1, \ldots, m_{\mathcal{T}}$, for some $m_{\mathcal{T}} \in \mathbb{N}$ and $C_{a s}, c_{a s}>0$, independent of $\kappa$ and $\mathcal{T}$, where $p_{\kappa}$ denotes the polynomial degree associated with element $\kappa, \kappa \in \mathcal{T}$.

The motivation for Definition 4.9 comes from the following result, which is derived in [31] and is instrumental in proving the inverse estimate stated in Lemma 4.11 below.

Lemma 4.10. Let $K$ be a shape-regular simplex. For each $v \in \mathcal{P}_{p}(K)$, there exists a simplex $\hat{\kappa} \subset K$, having the same shape as $K$ and faces parallel to the faces of $K$, with $\operatorname{dist}(\partial \hat{\kappa}, \partial K)>C_{a s} \operatorname{diam}(K) / p^{2}(>0)$, for some constant $C_{a s}>0$, independent of $v, K$ and $p$, such that

$$
\|v\|_{L_{2}(\hat{\kappa})} \geq \frac{1}{2}\|v\|_{L_{2}(K)} .
$$

We now recall from [21] the following inverse estimate for general polytopes, which is sharp with respect to degeneration of one or more of its $(d-k)$-dimensional, $k=1, \ldots, d-1$, facets.

Lemma 4.11. Let $\kappa \in \mathcal{T}, F \subset \partial \kappa$ denote one of its faces, and $\tilde{\mathcal{T}}$ be defined as in Definition 4.9. Then, for each $v \in \mathcal{P}_{p}(\kappa)$, we have the inverse estimate

$$
\|v\|_{L_{2}(F)}^{2} \leq C_{\mathrm{INV}}(p, \kappa, F) \frac{p^{2}|F|}{|\kappa|}\|v\|_{L_{2}(\kappa)}^{2},
$$


where

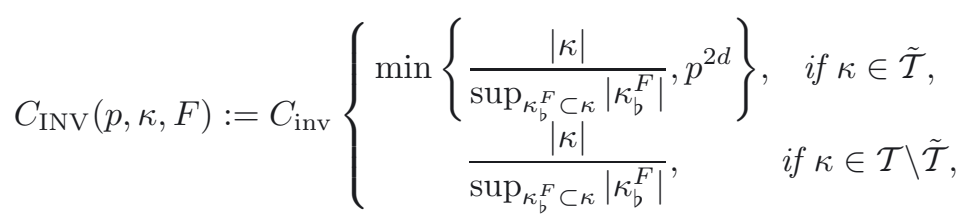

and $\kappa_{b}^{F} \in \mathcal{F}_{b}^{\kappa}$ is as in Definition 4.6. Furthermore, $C_{\mathrm{inv}}$ is a positive constant, which, if $\kappa \in \tilde{\mathcal{T}}$, depends on the shape regularity of the covering of $\kappa$ given in Definition 4.9 , but is always independent of $|\kappa| / \sup _{\kappa_{b}^{F} \subset \kappa}\left|\kappa_{b}^{F}\right|$ (and, therefore, of $|F|$ ), $p$, and $v$.

The following assumption plays a key role in deriving the extension of the standard inverse estimate for the $H^{1}$-(semi)norm.

Assumption 4.12. Every polytopic element $\kappa \in \mathcal{T} \backslash \tilde{\mathcal{T}}$, admits a sub-triangulation into at most $n_{\mathcal{T}}$ shaperegular simplices $\mathfrak{s}_{i}, i=1,2, \ldots, n_{\mathcal{T}}$, such that $\bar{\kappa}=\cup_{i=1}^{n_{\mathcal{T}}} \overline{\mathfrak{s}}_{i}$ and

$$
\left|\mathfrak{s}_{i}\right| \geq \hat{c}|\kappa|
$$

for all $i=1, \ldots, n_{\mathcal{T}}$, for some $n_{\mathcal{T}} \in \mathbb{N}$ and $\hat{c}>0$, independent of $\kappa$ and $\mathcal{T}$.

Lemma 4.13. Given Assumptions 4.1 and 4.12 are satisfied, for each $v \in \mathcal{P}_{p}(\kappa)$, the inverse estimate

$$
\|\nabla v\|_{L_{2}(\kappa)}^{2} \leq \tilde{C}_{\mathrm{inv}} \frac{p^{4}}{h_{\kappa}^{2}}\|v\|_{L_{2}(\kappa)}^{2}
$$

holds, with constant $\tilde{C}_{\mathrm{inv}}$ independent of the element diameter $h_{\kappa}$, the polynomial order $p_{\kappa}$, and the function $v$, but dependent on the shape regularity of the covering of $\kappa$, if $\kappa \in \tilde{\mathcal{T}}$, or the sub-triangulation of $\kappa$, if $\kappa \in \mathcal{T} \backslash \tilde{\mathcal{T}}$.

Proof. Here, we only consider the case when $\kappa \in \tilde{\mathcal{T}}$; for $\kappa \in \mathcal{T} \backslash \tilde{\mathcal{T}}$ the inverse inequality follows in a straightforward manner by exploiting the sub-triangulation introduced in Assumption 4.12, together with the standard inverse estimate applied to each sub-simplex. Given $\kappa \in \tilde{\mathcal{T}}$, we have a covering of $\kappa$ by shape-regular simplices $K_{j}, j=1, \ldots, m_{\mathcal{T}}$, with $\left|K_{j}\right| \geq c_{a s}|\kappa|, c f$. Definition 4.9. The shape-regularity Assumption 4.1, in conjunction with the trivial relation $h_{\kappa}^{d} \geq|\kappa| \geq \rho_{\kappa}^{d}$, yields

$$
h_{K_{j}}^{d} \geq\left|K_{j}\right| \geq c_{a s}|\kappa| \geq c_{a s} \rho_{\kappa}^{d} \geq c_{a s}\left(\frac{h_{\kappa}}{C_{\text {shape }}}\right)^{d},
$$

where $h_{K_{j}}$ denotes the diameter of $K_{j}$. Hence, $h_{K_{j}} \geq\left(c_{a s}\right)^{1 / d} h_{\kappa} / C_{\text {shape }}$. The standard inverse inequality applied on $K_{j}$ yields

$$
\|\nabla v\|_{L_{2}\left(K_{j}\right)} \leq C_{2} \frac{p^{2}}{h_{K_{j}}}\|v\|_{L_{2}\left(K_{j}\right)}
$$

where the positive constant $C_{2}$ is independent of $p, h_{K_{j}}$, and $v$; see [46] for details. Employing (4.6), (4.5), and Definition 4.9 again, we deduce that

$$
\begin{aligned}
\|\nabla v\|_{L_{2}(\kappa)}^{2} & \leq \sum_{j=1}^{m_{\mathcal{T}}}\|\nabla v\|_{L_{2}\left(K_{j}\right)}^{2} \\
& \leq C_{2} \sum_{j=1}^{m_{\mathcal{T}}} \frac{p^{4}}{h_{K_{j}}^{2}}\|v\|_{L_{2}\left(K_{j}\right)}^{2} \\
& \leq \frac{C_{2} C_{\text {shape }}^{2}}{\left(c_{a s}\right)^{2 / d}} \frac{p^{4}}{h_{\kappa}^{2}} \sum_{j=1}^{m_{\mathcal{T}}}\|v\|_{L_{2}\left(K_{j}\right)}^{2} .
\end{aligned}
$$


Let $\hat{\kappa}_{j} \subset K_{j}$ denote the simplex given by Lemma 4.10; then by Lemma 4.10 and Definition 4.9, we have

$$
\frac{1}{4}\|v\|_{L_{2}\left(K_{j}\right)}^{2} \leq\|v\|_{L_{2}\left(\hat{\kappa}_{j}\right)}^{2} \leq\|v\|_{L_{2}\left(K_{j} \cap \kappa\right)}^{2},
$$

since $\hat{\kappa}_{j} \subset \kappa$, and hence $\hat{\kappa}_{j} \subset K_{j} \cap \kappa \subset K_{j}$. Combining (4.7) and (4.8), we deduce that

$$
\|\nabla v\|_{L_{2}(\kappa)}^{2} \leq \frac{4 C_{2} C_{\mathrm{shape}}^{2} m_{\mathcal{T}}}{\left(c_{a s}\right)^{2 / d}} \frac{p^{4}}{h_{\kappa}^{2}}\|v\|_{L_{2}(\kappa)}^{2} \equiv \tilde{C}_{\mathrm{inv}} \frac{p^{4}}{h_{\kappa}^{2}}\|v\|_{L_{2}(\kappa)}^{2}
$$

as required.

Remark 4.14. We point out that Assumption 4.1, which imposes the shape regularity of the mesh $\mathcal{T}$, is only needed for the proof of Lemma 4.13; this result extends the classical inverse estimate, bounding the $H^{1}$-seminorm of a polynomial function with its $L_{2}$-norm, to polytopic elements. We note, however, that such inverse estimates depend on the shape regularity of the elements, even in the case of simplicial elements, $c f$. [49]. An alternative $h p$-version inverse inequality, analogous to Lemma 4.13, with explicit dependence on the shape-regularity, is derived in [6].

\section{A PRIORI ERROR BOUND FOR THE DGFEM}

The a priori error analysis will employ an inconsistent formulation of the diffusion part of the bilinear form, cf. [43], for example. We define, for all $u, v \in \mathcal{S}:=H^{1}(\Omega)+S_{\mathcal{T}}^{\mathrm{p}}$, the bilinear form

$$
\tilde{B}(u, v):=B_{\operatorname{ar}}(u, v)+\tilde{B}_{\mathrm{d}}(u, v),
$$

where

$$
\begin{aligned}
\tilde{B}_{\mathrm{d}}(u, v):= & \sum_{\kappa \in \mathcal{T}} \int_{\kappa} a \nabla u \cdot \nabla v \mathrm{~d} x+\int_{\Gamma_{\mathrm{int}} \cup \Gamma_{\mathrm{D}}} \sigma \llbracket u \rrbracket \cdot \llbracket v \rrbracket \mathrm{d} s \\
& -\int_{\Gamma_{\mathrm{int}} \cup \Gamma_{\mathrm{D}}}\left(\left\{\left\{a \Pi_{2}\left(\nabla_{h} u\right)\right\}\right] \cdot \llbracket v \rrbracket+\left\{\left\{a \Pi_{2}\left(\nabla_{h} v\right)\right\}\right\} \cdot \llbracket u \rrbracket\right) \mathrm{d} s,
\end{aligned}
$$

and the linear functional $\tilde{\ell}: S_{\mathcal{T}}^{\mathbf{p}} \rightarrow \mathbb{R}$ by

$$
\begin{aligned}
\tilde{\ell}(v):= & \sum_{\kappa \in \mathcal{T}} \int_{\kappa} f v \mathrm{~d} x-\sum_{\kappa \in \mathcal{T}} \int_{\partial_{-} \kappa \cap\left(\Gamma_{\mathrm{D}} \cup \Gamma_{-}\right)}(\mathbf{b} \cdot \mathbf{n}) g_{\mathrm{D}} v^{+} \mathrm{d} s \\
& -\int_{\Gamma_{\mathrm{D}}} g_{\mathrm{D}}\left(a \boldsymbol{\Pi}_{2}\left(\nabla_{h} v\right) \cdot \mathbf{n}-\sigma v\right) \mathrm{d} s+\int_{\Gamma_{\mathrm{N}}} g_{\mathrm{N}} v \mathrm{~d} s .
\end{aligned}
$$

Here, $\boldsymbol{\Pi}_{2}:\left[L_{2}(\Omega)\right]^{d} \rightarrow\left[S_{\mathcal{T}}^{\mathbf{p}-1}\right]^{d}$ denotes the $L_{2}$-projection onto the finite element space $\left[S_{\mathcal{T}}^{\mathbf{p}-\mathbf{1}}\right]^{d}$. We then rewrite the discrete problem with the inconsistent formulation in the equivalent form: find $u_{h} \in S_{\mathcal{T}}^{\mathbf{p}}$ such that

$$
\tilde{B}\left(u_{h}, v_{h}\right)=\tilde{l}\left(v_{h}\right) \quad \forall v_{h} \in S_{\mathcal{T}}^{\mathbf{p}} .
$$

In view of the error analysis, we introduce the DGFEM-norm $\mid\|\cdot\|_{\mathrm{DG}}$ as the sum of two parts as follows:

$$
\left\|v \left|\left\|_{\mathrm{DG}}^{2}:=\left|\|v\|_{\mathrm{ar}}^{2}+\|\| v\right|\right\|_{\mathrm{d}}^{2},\right.\right.
$$

where

$$
\|v\|_{\text {ar }}^{2}:=\sum_{\kappa \in \mathcal{T}}\left(\left\|c_{0} v\right\|_{L_{2}(\kappa)}^{2}+\frac{1}{2}\left\|v^{+}\right\|_{\partial_{-} \kappa \cap\left(\Gamma_{\mathrm{D}} \cup \Gamma_{-}\right)}^{2}+\frac{1}{2}\left\|v^{+}-v^{-}\right\|_{\partial_{-} \kappa \backslash \Gamma}^{2}+\frac{1}{2}\left\|v^{+}\right\|_{\partial_{+} \kappa \cap \Gamma}^{2}\right),
$$


with $c_{0}$ as in (2.5), and

$$
\|v\|_{\mathrm{d}}^{2}:=\sum_{\kappa \in \mathcal{T}}\|\sqrt{a} \nabla v\|_{L_{2}(\kappa)}^{2}+\int_{\Gamma_{\mathrm{int}} \cup \Gamma_{\mathrm{D}}} \sigma|\llbracket v \rrbracket|^{2} \mathrm{~d} s .
$$

Here, $\|\cdot\|_{\tau}, \tau \subset \partial \kappa$, denotes the (semi)norm associated with the (semi)inner product $(v, w)_{\tau}=\int_{\tau}|\mathbf{b} \cdot \mathbf{n}| v w \mathrm{~d} s$.

The following relation holds:

$$
B_{\operatorname{ar}}(v, v)=\|\| v \|_{\mathrm{ar}}^{2}
$$

for all $v \in \mathcal{S}$, cf. [36]. The continuity and coercivity of the inconsistent diffusion bilinear form $\tilde{B}_{\mathrm{d}}(\cdot, \cdot)$, with respect to the DGFEM-norm $\|\cdot\|_{\mathrm{d}}$, is established by the following lemma.

Lemma 5.1. Let $\sigma: \Gamma \backslash \Gamma_{\mathrm{N}} \rightarrow \mathbb{R}_{+}$be defined facewise by

$$
\sigma(x):= \begin{cases}C_{\sigma} \max _{\kappa \in\left\{\kappa_{1}, \kappa_{2}\right\}}\left\{C_{\mathrm{INV}}\left(p_{\kappa}, \kappa, F\right) \frac{\bar{a}_{\kappa} p_{\kappa}^{2}|F|}{|\kappa|}\right\}, & x \in F \subset \Gamma_{\mathrm{int}}, F=\partial \kappa_{1} \cap \partial \kappa_{2}, \\ C_{\sigma} C_{\mathrm{INV}}\left(p_{\kappa}, \kappa, F\right) \frac{\bar{a}_{\kappa} p_{\kappa}^{2}|F|}{|\kappa|}, & x \in F \subset \Gamma_{\mathrm{D}}, F=\partial \kappa \cap \Gamma_{\mathrm{D}},\end{cases}
$$

with $C_{\sigma}>0$ large enough, and independent of $p_{\kappa},|F|$, and $|\kappa|$; here $C_{\mathrm{INV}}$ is defined as in Lemma 4.11. Then, given Assumption 4.2 holds, we have that

$$
\tilde{B}_{\mathrm{d}}(v, v) \geq C_{\mathrm{d}}^{\text {coer }} \mid\|v\|_{\mathrm{d}}^{2} \quad \text { for all } \quad v \in \mathcal{S},
$$

and

$$
\tilde{B}_{\mathrm{d}}(w, v) \leq C_{\mathrm{d}}^{\text {cont }}\left|\|w\|_{\mathrm{d}}\right|\|v\|_{\mathrm{d}} \quad \text { for all } \quad w, v \in \mathcal{S}
$$

where $C_{\mathrm{d}}^{\mathrm{cont}}$ and $C_{\mathrm{d}}^{\mathrm{coer}}$ are positive constants independent of the discretization parameters.

Proof. The proof can be viewed as an extension of the analogous result derived in [21] for the Poisson equation, to a second-order elliptic PDE with general positive semidefinite diffusion tensor $a$; for details, we refer to [32].

\subsection{Inf-Sup condition}

The $h p$-version a priori error analysis presented in [36] relies on the derivation of optimal $h p$-approximation results for the trace of the local $L_{2}$-projection operator on a given face of an element $\kappa$ in the finite element mesh $\mathcal{T}$, cf. also [22] for analogous results on simplices. Due to the lack of analogous $h p$-approximation results for the local $L_{2}$-projection operator on polytopic elements, it is not possible to directly generalise the analysis from [36] to meshes consisting of such elements. To address this issue we prove an inf-sup condition for the inconsistent bilinear form $\tilde{B}(\cdot, \cdot)$, with respect to the following streamline DGFEM-norm.

Definition 5.2. The streamline DGFEM-norm is defined by:

$$
\|v \mid\|_{\mathrm{s}}^{2}:=\|v\|_{\mathrm{DG}}^{2}+\sum_{\kappa \in \mathcal{T}} \tau_{\kappa}\|\mathbf{b} \cdot \nabla v\|_{L_{2}(\kappa)}^{2},
$$

where

$$
\tau_{\kappa}:=\min \left\{\frac{1}{\|\mathbf{b}\|_{L_{\infty}(\kappa)}}, \frac{1}{\tilde{\sigma}_{\kappa}}\right\} \frac{h_{\kappa}^{\perp}}{p_{\kappa}^{2}} \quad \forall \kappa \in \mathcal{T}
$$

for $p_{\kappa} \geq 1$, and $\tilde{\sigma}_{\kappa}$ is given by

$$
\tilde{\sigma}_{\kappa}:=C_{\sigma} \max _{F \subset \partial \kappa}\left\{\max _{\substack{\tilde{\kappa} \in\left\{\kappa, \kappa^{\prime}\right\} \\ F \subset \partial \kappa \cap \partial \kappa^{\prime}}}\left\{C_{\mathrm{inv}} \frac{\bar{a}_{\tilde{\kappa}} p_{\tilde{\kappa}}^{2}}{h_{\tilde{\kappa}}^{\perp}} d\right\}\right\} \quad \forall \kappa \subset \mathcal{T}, \quad d=2,3,
$$


where $C_{\mathrm{inv}}$ is defined as in Lemma 4.11 . The constant $\tilde{\sigma}_{\kappa}$ may be zero locally where $\bar{a}_{\kappa}=0$; in this case it is understood that $\tau_{\kappa}$ takes the value of the first term in (5.10). Further, the mesh parameter $h_{\kappa}^{\perp}$ is defined as follows:

$$
h_{\kappa}^{\perp}:=\min _{F \subset \partial \kappa} \frac{\sup _{\kappa_{b}^{F} \subset \kappa}\left|\kappa_{b}^{F}\right|}{|F|} d \quad \forall \kappa \in \mathcal{T}, \quad d=2,3,
$$

with $\kappa_{b}^{F}$ as in Definition 4.6. We further deduce the relation

$$
h_{\kappa}^{\perp} \leq h_{\kappa} .
$$

Remark 5.3. We recall from Definition 4.6 that $\kappa_{b}^{F}$ denotes the family of simplices contained in $\kappa$ and sharing a face $F$ with $\kappa$. From the geometrical property of $d$-dimensional simplices, it is easy to see that $h_{\kappa}^{\perp}$ is the minimum over all faces $F, F \subset \partial \kappa$, of the maximum of the set of all heights of the $d$-dimensional simplices $\kappa_{b}^{F}$ sharing a $(d-1)$-dimensional face $F$ with $\kappa$.

Remark 5.4. With a mild loss of generality, the case $p_{\kappa}=0$, relevant to the hyperbolic regime, is excluded from Definition 5.2 and throughout this paper. However, if the underlying problem is strictly hyperbolic and $p_{\kappa}=0$ is selected for all $\kappa \in \mathcal{T}$, then the streamline DGFEM-norm reduces to the advection-reaction DGFEM-norm $\||| \cdot\|_{\text {ar }}$ defined in (5.3); in this setting, the proceeding analysis is trivial.

By employing the definition of $h_{\kappa}^{\perp}$, together with an upper bound on the constant $C_{\mathrm{INV}}(p, \kappa, F)$ defined in Lemma 4.11, the inverse estimate (4.3) can be written in the following manner. For each $v \in \mathcal{P}_{p}(\kappa), F \subset \partial \kappa$, we have

$$
\begin{aligned}
\|v\|_{L_{2}(F)}^{2} & \leq C_{\mathrm{INV}}(p, \kappa, F) \frac{p^{2}|F|}{|\kappa|}\|v\|_{L_{2}(\kappa)}^{2} \\
& \leq C_{\operatorname{inv}} \frac{|\kappa|}{\sup _{\kappa_{\mathrm{b}}^{F} \subset \kappa}\left|\kappa_{b}^{F}\right|} \frac{p^{2}|F|}{|\kappa|}\|v\|_{L_{2}(\kappa)}^{2} \leq C_{\mathrm{inv}} \frac{p^{2}}{h_{\kappa}^{\perp}} d\|v\|_{L_{2}(\kappa)}^{2} .
\end{aligned}
$$

Further, from the definition of $\left.\sigma\right|_{F}$ given in (5.6), in conjunction with the definition of $h_{\kappa}^{\perp}, c f$. (5.12), we deduce the bound

$$
\tilde{\sigma}_{\kappa} \geq\left.\sigma\right|_{F}, \quad F \subset \partial \kappa \forall \kappa \in \mathcal{T}
$$

For the reminder of this article we assume the following standard condition on $\mathbf{b}$ :

$$
\mathbf{b} \cdot \nabla_{h} \xi \in S_{\mathcal{T}}^{\mathbf{p}} \quad \forall \xi \in S_{\mathcal{T}}^{\mathbf{p}},
$$

cf. [36]. Under the above assumption, we prove the inf-sup condition for the bilinear form $\tilde{B}(\cdot, \cdot)$, with respect to the streamline DGFEM-norm (5.9).

Theorem 5.5. Given Assumptions 4.1, 4.2, and 4.12 hold, there exists a positive constant $\Lambda_{s}$, independent of the mesh size $h$ and the polynomial degree $p$, such that:

$$
\inf _{\nu \in S_{\mathcal{T}}^{\mathbf{P}} \backslash\{0\}} \sup _{\mu \in S_{\mathcal{T}}^{\mathbf{p}} \backslash\{0\}} \frac{\tilde{B}(\nu, \mu)}{\left\|\nu \left|\left\|_{\mathrm{s}} \mid\right\| \mu \|_{\mathrm{s}}\right.\right.} \geq \Lambda_{s},
$$

where the discontinuity-penalization parameter $\sigma$ is as defined in (5.6).

Proof. For all $\nu \in S_{\mathcal{T}}^{\text {p }}$, we select $\mu:=\nu+\alpha \nu_{s},\left.\nu_{s}\right|_{\kappa}=\tau_{\kappa} \mathbf{b} \cdot \nabla \nu$ for all $\kappa \in \mathcal{T}$, where $\alpha$ is a positive real number, chosen sufficiently small, $c f$. (5.32) below. By (5.16), we note that $\mu \in S_{\mathcal{T}}^{\mathbf{p}}$; the theorem now follows from the two bounds:

$$
\left\|\left|\left\|\mu \left|\left\|_{\mathrm{s}} \leq C^{*}\left|\|\nu \mid\|_{\mathrm{s}}\right.\right.\right.\right.\right.\right.
$$


and

$$
\tilde{B}(\nu, \mu) \geq C_{*}\left|\|\nu \mid\|_{\mathrm{s}}^{2}\right.
$$

with $\Lambda_{s}=C_{*} / C^{*}$, where $C^{*}$ and $C_{*}$ are positive constants, independent of $h$ and $p$.

We begin by proving (5.18). We first bound each term arising in the norm ||$|\cdot|||_{\text {ar }}$ of $\nu_{s}$, where $\left.\nu_{s}\right|_{\kappa}=\tau_{\kappa} \mathbf{b} \cdot \nabla \nu$, $\kappa \in \mathcal{T}$. Employing Lemma 4.13 together with (5.10), the lower bound on $c_{0}$ given in (2.5), and inequality (5.13), gives

$$
\begin{aligned}
\sum_{\kappa \in \mathcal{T}}\left\|c_{0} \nu_{s}\right\|_{L_{2}(\kappa)}^{2} & \leq\left\|c_{0}\right\|_{L_{\infty}(\Omega)}^{2} \sum_{\kappa \in \mathcal{T}} \tau_{\kappa}^{2}\|\mathbf{b} \cdot \nabla \nu\|_{L_{2}(\kappa)}^{2} \\
& \leq\left\|c_{0}\right\|_{L_{\infty}(\Omega)}^{2} \tilde{C}_{\mathrm{inv}} \sum_{\kappa \in \mathcal{T}} \tau_{\kappa}^{2} \frac{p_{\kappa}^{4}\|\mathbf{b}\|_{L_{\infty}(\kappa)}^{2}\|\nu\|_{L_{2}(\kappa)}^{2}}{h_{\kappa}^{2}} \\
& \leq\left\|c_{0}\right\|_{L_{\infty}(\Omega)}^{2} \frac{\tilde{C}_{\mathrm{inv}}}{\gamma_{0}} \sum_{\kappa \in \mathcal{T}}\left\|c_{0} \nu\right\|_{L_{2}(\kappa)}^{2} \leq C_{1}\|\nu\|_{\mathrm{s}}^{2} .
\end{aligned}
$$

Using the inverse estimate (5.14), we deduce that

$$
\begin{aligned}
\sum_{\kappa \in \mathcal{T}}\left(\frac{1}{2}\left\|\nu_{s}^{+}\right\|_{\partial_{-} \kappa}^{2}\right. & \left(\Gamma_{\mathrm{D}} \cup \Gamma_{-}\right) \\
\leq & \left.\sum_{\kappa \in \mathcal{T}}\|\mathbf{b}\|_{L_{\infty}(\kappa)} \tau_{\kappa}^{2} \sum_{F \subset \partial \kappa}\left\|\mathbf{b} \cdot \nu_{s}^{+}-\nu_{s}^{-}\right\|_{\partial_{-} \kappa \backslash \Gamma}^{2}+\frac{1}{2}\left\|\nu_{L_{2}(F)}^{+}\right\|_{\partial_{+} \kappa \cap \Gamma}^{2}\right) \\
& \leq C_{F} C_{\text {inv }} d \sum_{\kappa \in \mathcal{T}} \tau_{\kappa} \frac{p_{\kappa}^{2}\|\mathbf{b}\|_{L_{\infty}(\kappa)}}{h_{\kappa}^{\perp}}\left(\tau_{\kappa}\|\mathbf{b} \cdot \nabla \nu\|_{L_{2}(\kappa)}^{2}\right) \leq C_{2}\|\nu\|_{\mathrm{s}}^{2} .
\end{aligned}
$$

Similarly, employing Lemma 4.13, the streamline diffusion term, $c f .(5.9)$, can be bounded as follows:

$$
\begin{aligned}
\sum_{\kappa \in \mathcal{T}} \tau_{\kappa}\left\|\mathbf{b} \cdot \nabla \nu_{s}\right\|_{L_{2}(\kappa)}^{2} & \leq \sum_{\kappa \in \mathcal{T}} \tau_{\kappa}\|\mathbf{b}\|_{L_{\infty}(\kappa)}^{2}\left(\tau_{\kappa}^{2}\|\nabla(\mathbf{b} \cdot \nabla \nu)\|_{L_{2}(\kappa)}^{2}\right) \\
& \leq \sum_{\kappa \in \mathcal{T}} \tilde{C}_{\mathrm{inv}} \tau_{\kappa}^{2} \frac{p_{\kappa}^{4}\|\mathbf{b}\|_{L_{\infty}(\kappa)}^{2}}{h_{\kappa}^{2}}\left(\tau_{\kappa}\|\mathbf{b} \cdot \nabla \nu\|_{L_{2}(\kappa)}^{2}\right) \\
& \leq \sum_{\kappa \in \mathcal{T}} \tilde{C}_{\mathrm{inv}}\left(\tau_{\kappa}\|\mathbf{b} \cdot \nabla \nu\|_{L_{2}(\kappa)}^{2}\right) \leq C_{3}\|\nu\|_{\mathrm{s}}^{2}
\end{aligned}
$$

here, we have again exploited a bound on $\tau_{\kappa}, \kappa \in \mathcal{T}$, and (5.13), cf. above.

Secondly, we consider the diffusion component $\|\cdot\|_{\mathrm{d}}$ of the streamline DGFEM-norm of $\nu_{s}$. This time, the second term on the right hand side of (5.10) is used as an upper bound on $\tau_{\kappa}, \kappa \in \mathcal{T}$. Thereby, employing Lemma 4.13, the definition of $\tilde{\sigma}_{\kappa}$ in (5.11), and (5.13), we get

$$
\begin{aligned}
\sum_{\kappa \in \mathcal{T}}\left\|\sqrt{a} \nabla \nu_{s}\right\|_{L_{2}(\kappa)}^{2} & \leq \sum_{\kappa \in \mathcal{T}} \bar{a}_{\kappa} \tau_{\kappa}^{2}\|\nabla(\mathbf{b} \cdot \nabla \nu)\|_{L_{2}(\kappa)}^{2} \\
& \leq \sum_{\kappa \in \mathcal{T}} \tilde{C}_{\mathrm{inv}} \tau_{\kappa} \frac{\bar{a}_{\kappa} p_{\kappa}^{4}}{h_{\kappa}^{2}}\left(\tau_{\kappa}\|\mathbf{b} \cdot \nabla \nu\|_{L_{2}(\kappa)}^{2}\right) \\
& \leq \sum_{\kappa \in \mathcal{T}} \tilde{C}_{\mathrm{inv}} \frac{\bar{a}_{\kappa} p_{\kappa}^{2}}{\tilde{\sigma}_{\kappa} h_{\kappa}}\left(\tau_{\kappa}\|\mathbf{b} \cdot \nabla \nu\|_{L_{2}(\kappa)}^{2}\right) \\
& \leq \frac{\tilde{C}_{\mathrm{inv}}}{C_{\sigma} C_{\mathrm{inv}} d} \sum_{\kappa \in \mathcal{T}} \tau_{\kappa}\|\mathbf{b} \cdot \nabla \nu\|_{L_{2}(\kappa)}^{2} \equiv C_{4} \sum_{\kappa \in \mathcal{T}} \tau_{\kappa}\|\mathbf{b} \cdot \nabla \nu\|_{L_{2}(\kappa)}^{2} \leq C_{4}\|\nu\|_{\mathrm{s}}^{2} .
\end{aligned}
$$


Finally, employing (5.14) and noting that $\left.\sigma\right|_{F} \leq \tilde{\sigma}_{\kappa}$ for $F \subset \partial \kappa, \kappa \in \mathcal{T}$, gives

$$
\begin{aligned}
\int_{\Gamma_{\mathrm{int}} \cup \Gamma_{D}} \sigma\left|\llbracket \nu_{s} \rrbracket\right|^{2} \mathrm{~d} s & \leq 2 \sum_{\kappa \in \mathcal{T}} \tau_{\kappa}^{2} \sum_{F \subset \partial \kappa \cap\left(\Gamma_{\mathrm{int}} \cup \Gamma_{D}\right)} \sigma\left\|\mathbf{b} \cdot \nabla_{h} \nu\right\|_{L_{2}(F)}^{2} \\
& \leq 2 C_{F} C_{\mathrm{inv}} d \sum_{\kappa \in \mathcal{T}} \tau_{\kappa} \frac{\tilde{\sigma}_{\kappa} p_{\kappa}^{2}}{h_{\kappa}^{\perp}}\left(\tau_{\kappa}\|\mathbf{b} \cdot \nabla \nu\|_{L_{2}(\kappa)}^{2}\right) \\
& \leq C_{5} \sum_{\kappa \in \mathcal{T}}\left(\tau_{\kappa}\|\mathbf{b} \cdot \nabla \nu\|_{L_{2}(\kappa)}^{2}\right) \leq C_{5}\|\nu\|_{\mathrm{s}}^{2} .
\end{aligned}
$$

Combining the above bounds, we deduce that

$$
\left\|\nu_{s}\right\|_{\mathrm{s}} \leq \hat{C} \mid\|\nu\|_{\mathrm{s}}
$$

where $\hat{C}=\sqrt{C_{1}+C_{2}+C_{3}+C_{4}+C_{5}}$. Exploiting the triangle inequality, we have that

$$
\left\|\mu \mu \left|\left\|_{\mathrm{s}} \leq\left|\left\|\nu \left|\left\|_{\mathrm{s}}+\alpha\left|\left\|\nu _ { \mathrm { s } } \left|\left\|_{\mathrm{s}} \leq(1+\alpha \hat{C})\right\|\left\|\nu\left|\left\|_{\mathrm{s}} \equiv C^{*}(\alpha)\right\| \nu\right|\right\|_{\mathrm{s}}\right.\right.\right.\right.\right.\right.\right.\right.\right.\right.
$$

which gives the desired bound stated in (5.18).

Next we prove (5.19). To this end, we observe that $\tilde{B}(\nu, \mu)=\tilde{B}(\nu, \nu)+\alpha \tilde{B}\left(\nu, \nu_{s}\right)$. Considering the second term $\tilde{B}\left(\nu, \nu_{s}\right)$ first, we note that the advection-reaction part of the bilinear form $B_{\mathrm{ar}}\left(\nu, \nu_{s}\right)$ is given by

$$
\begin{aligned}
B_{\mathrm{ar}}\left(\nu, \nu_{s}\right)= & \sum_{\kappa \in \mathcal{T}} \int_{\kappa} \tau_{\kappa}(\mathbf{b} \cdot \nabla \nu)^{2}+c \nu\left(\tau_{\kappa} \mathbf{b} \cdot \nabla \nu\right) \mathrm{d} x \\
& -\int_{\partial_{-} \backslash \Gamma}(\mathbf{b} \cdot \mathbf{n})\lfloor\nu\rfloor\left(\tau_{\kappa} \mathbf{b} \cdot \nabla \nu\right)^{+} \mathrm{d} s-\int_{\partial_{-} \kappa \cap\left(\Gamma_{\mathrm{D}} \cup \Gamma_{-}\right)}(\mathbf{b} \cdot \mathbf{n}) \nu^{+}\left(\tau_{\kappa} \mathbf{b} \cdot \nabla \nu\right)^{+} \mathrm{d} s .
\end{aligned}
$$

Employing Lemma 4.13, together with the lower bound on $c_{0}^{2}$ given in (2.5), the second term in (5.27) may be bounded as follows:

$$
\begin{aligned}
\left|\sum_{\kappa \in \mathcal{T}} \int_{\kappa} c \nu\left(\tau_{\kappa} \mathbf{b} \cdot \nabla \nu\right) \mathrm{d} x\right| & \leq \sum_{\kappa \in \mathcal{T}}\|c\|_{L_{\infty}(\Omega)}\|\nu\|_{L_{2}(\kappa)}\left\|\tau_{\kappa} \mathbf{b} \cdot \nabla \nu\right\|_{L_{2}(\kappa)} \\
& \leq \sum_{\kappa \in \mathcal{T}}\|c\|_{L_{\infty}(\Omega)}\|\nu\|_{L_{2}(\kappa)}\left(\tilde{C}_{\mathrm{inv}}^{1 / 2} \tau_{\kappa} \frac{p_{\kappa}^{2}\|\mathbf{b}\|_{L_{\infty}(\kappa)}}{h_{\kappa}}\|\nu\|_{L_{2}(\kappa)}\right) \\
& \leq \sum_{\kappa \in \mathcal{T}} \frac{\tilde{C}_{\mathrm{inv}}^{1 / 2}\|c\|_{L_{\infty}(\Omega)}}{\gamma_{0}}\left\|c_{0} \nu\right\|_{L_{2}(\kappa)}^{2} .
\end{aligned}
$$


To estimate the boundary terms present in (5.27), we exploit the inverse estimate (5.14), the definition of $\tau_{\kappa}$ given in (5.10), together with the Cauchy-Schwarz inequality; thereby, we get

$$
\begin{aligned}
\mid \sum_{\kappa \in \mathcal{T}} & \left(\int_{\partial_{-} \kappa \backslash \Gamma}(\mathbf{b} \cdot \mathbf{n})\lfloor\nu\rfloor\left(\tau_{\kappa} \mathbf{b} \cdot \nabla \nu\right)^{+} \mathrm{d} s+\int_{\partial_{-} \kappa \cap\left(\Gamma_{\mathrm{D}} \cup \Gamma_{-}\right)}(\mathbf{b} \cdot \mathbf{n}) \nu^{+}\left(\tau_{\kappa} \mathbf{b} \cdot \nabla_{h} \nu\right)^{+} \mathrm{d} s\right) \mid \\
\leq & \sum_{\kappa \in \mathcal{T}}\left\|\nu^{+}-\nu^{-}\right\| \partial_{\partial_{-}} \backslash \Gamma\left(\sum_{F \subset \partial_{-} \backslash \Gamma}\|\mathbf{b}\|_{L_{\infty}(\kappa)}^{1 / 2} \tau_{\kappa}\left\|\mathbf{b} \cdot \nabla_{h} \nu\right\|_{L_{2}(F)}\right) \\
& +\sum_{\kappa \in \mathcal{T}}\left\|\nu^{+}\right\|_{\partial_{-} \kappa \cap\left(\Gamma_{\mathrm{D}} \cup \Gamma_{-}\right)}\left(\sum_{F \subset \partial_{-} \kappa \cap\left(\Gamma_{\mathrm{D}} \cup \Gamma_{-}\right)}\|\mathbf{b}\|_{L_{\infty}(\kappa)}^{1 / 2} \tau_{\kappa}\left\|\mathbf{b} \cdot \nabla_{h} \nu\right\|_{L_{2}(F)}\right) \\
\leq & C_{F}^{2} C_{\mathrm{inv}} d\left(\sum_{\kappa \in \mathcal{T}}\left\|\nu^{+} \nu^{-}\right\|_{\partial_{-} \kappa \backslash \Gamma}^{2}+\left\|\nu^{+}\right\|_{\partial_{-} \kappa \cap\left(\Gamma_{\mathrm{D}} \cup \Gamma_{-}\right)}^{2}\right)+\sum_{\kappa \in \mathcal{T}} \frac{\tau_{\kappa}}{4}\|\mathbf{b} \cdot \nabla \nu\|_{L_{2}(\kappa)}^{2} \\
\leq & C_{F}^{2} C_{\mathrm{inv}} d \sum_{\kappa \in \mathcal{T}}\left(\left\|\nu^{+}-\nu^{-}\right\|_{\partial_{-} \kappa \backslash \Gamma}^{2}+\left\|\nu^{+}\right\|_{\partial_{-} \kappa \cap\left(\Gamma_{\mathrm{D}} \cup \Gamma_{-}\right)}^{2}+\left\|\nu^{+}\right\|_{\partial_{+} \kappa \cap \Gamma}^{2}\right)+\sum_{\kappa \in \mathcal{T}} \frac{\tau_{\kappa}}{4}\|\mathbf{b} \cdot \nabla \nu\|_{L_{2}(\kappa)}^{2} .
\end{aligned}
$$

Using (5.5), together with the bounds (5.28) and (5.29), we deduce that

$$
\begin{aligned}
B_{\mathrm{ar}}(\nu, \mu) \geq & \left(1-\frac{\alpha \tilde{C}_{\mathrm{inv}}^{1 / 2}\|c\|_{L_{\infty}(\Omega)}}{\gamma_{0}}\right) \sum_{\kappa \in \mathcal{T}}\left\|c_{0} \nu\right\|_{L_{2}(\kappa)}^{2}+\alpha \sum_{\kappa \in \mathcal{T}}\left(\tau_{\kappa}-\frac{\tau_{\kappa}}{4}\right)\|\mathbf{b} \cdot \nabla \nu\|_{L_{2}(\kappa)}^{2} . \\
& +\left(\frac{1}{2}-\alpha C_{F}^{2} C_{\mathrm{inv}} d\right) \sum_{\kappa \in \mathcal{T}}\left(\left\|\nu^{+}-\nu^{-}\right\|_{\partial_{-} \kappa \backslash \Gamma}^{2}+\left\|\nu^{+}\right\|_{\partial_{-} \kappa \cap\left(\Gamma_{\mathrm{D}} \cup \Gamma_{-}\right)}^{2}+\left\|\nu^{+}\right\|_{\partial_{+} \kappa \cap \Gamma}^{2}\right) .
\end{aligned}
$$

Next, we consider the diffusion part of bilinear form, i.e., $\tilde{B}_{\mathrm{d}}\left(\nu, \nu_{s}\right)$. From the continuity of $\tilde{B}_{\mathrm{d}}(\cdot, \cdot)$ stated in $(5.8)$, together with the bounds given in (5.23) and (5.24), we get

$$
\begin{aligned}
\tilde{B}_{\mathrm{d}}\left(\nu, \nu_{s}\right) & \leq C_{\mathrm{d}}^{\mathrm{cont}}\left|\left\|\nu\left|\left\|_{\mathrm{d}}\right\| \nu_{s}\right|\right\|_{\mathrm{d}} \leq C_{\mathrm{d}}^{\mathrm{cont}}\right|\|\nu\|_{\mathrm{d}} \sqrt{C_{4}+C_{5}}\left(\sum_{\kappa \in \mathcal{T}} \tau_{\kappa}\|\mathbf{b} \cdot \nabla \nu\|_{L_{2}(\kappa)}^{2}\right)^{1 / 2} \\
& \leq\left(C_{\mathrm{d}}^{\mathrm{cont}}\right)^{2}\left(C_{4}+C_{5}\right) \mid\|\nu\|_{\mathrm{d}}^{2}+\sum_{\kappa \in \mathcal{T}} \frac{\tau_{\kappa}}{4}\|\mathbf{b} \cdot \nabla \nu\|_{L_{2}(\kappa)}^{2} .
\end{aligned}
$$

Exploiting the coercivity of the bilinear form $\tilde{B}_{d}(\cdot, \cdot), c f .(5.7)$, gives

$$
\tilde{B}_{\mathrm{d}}(\nu, \mu) \geq\left(C_{\mathrm{d}}^{\text {coer }}-\alpha\left(C_{\mathrm{d}}^{\text {cont }}\right)^{2}\left(C_{4}+C_{5}\right)\right)\|\nu\|_{\mathrm{d}}^{2}-\alpha \sum_{\kappa \in \mathcal{T}} \frac{\tau_{\kappa}}{4}\|\mathbf{b} \cdot \nabla \nu\|_{L_{2}(\kappa)}^{2} .
$$

Finally, combining (5.30) and (5.31), the following bound holds:

$$
\begin{aligned}
\tilde{B}(\nu, \mu)= & B_{\mathrm{ar}}(\nu, \mu)+\tilde{B}_{\mathrm{d}}(\nu, \mu) \\
\geq & \left(1-\frac{\alpha \tilde{C}_{\mathrm{inv}}^{1 / 2}\|c\|_{L_{\infty}(\Omega)}}{\gamma_{0}}\right) \sum_{\kappa \in \mathcal{T}}\left\|c_{0} \nu\right\|_{L_{2}(\kappa)}^{2}+\alpha \sum_{\kappa \in \mathcal{T}}\left(\tau_{\kappa}-\frac{\tau_{\kappa}}{4}-\frac{\tau_{\kappa}}{4}\right)\|\mathbf{b} \cdot \nabla \nu\|_{L_{2}(\kappa)}^{2} . \\
& +\left(\frac{1}{2}-\alpha C_{F}^{2} C_{\mathrm{inv}} d\right) \sum_{\kappa \in \mathcal{T}}\left(\left\|\nu^{+}-\nu^{-}\right\|_{\partial_{-} \varsigma \Gamma \Gamma}^{2}+\left\|\nu^{+}\right\|_{\partial_{-} \kappa \cap\left(\Gamma_{\mathrm{D}} \cup \Gamma_{-}\right)}^{2}+\left\|\nu^{+}\right\|_{\partial_{+} \kappa \cap \Gamma}^{2}\right) . \\
& +\left(C_{\mathrm{d}}^{\mathrm{coer}}-\alpha\left(C_{\mathrm{d}}^{\mathrm{cont}}\right)^{2}\left(C_{4}+C_{5}\right)\right)\left(\sum_{\kappa \in \mathcal{T}}\|\sqrt{a} \nabla \nu\|_{L_{2}(\kappa)}^{2}+\int_{\Gamma_{\mathrm{int}} \cup \Gamma_{\mathrm{D}}} \sigma|\llbracket \nu \||^{2} \mathrm{~d} s\right) .
\end{aligned}
$$


The coefficients in front of the norms arising on the right hand side of the above bound are all positive for sufficient small $\alpha$, namely if

$$
\alpha<\min \left\{\frac{\gamma_{0}}{\tilde{C}_{\mathrm{inv}}^{1 / 2}\|c\|_{L_{\infty}(\Omega)}}, \frac{1}{2 C_{F}^{2} C_{\mathrm{inv}} d}, \frac{C_{\mathrm{d}}^{\text {coer }}}{\left(C_{\mathrm{d}}^{\text {cont }}\right)^{2}\left(C_{4}+C_{5}\right)}\right\} .
$$

Since the constants in (5.32) are independent of the discretization parameters, we conclude that (5.19) holds as long as $\alpha$ is chosen according to (5.32).

Remark 5.6. Theorem 5.5 extends the analogous result derived for DGFEMs on meshes comprising of simplices presented in $[19,20]$ to general polytopic elements. It also improves those results in the sense that here the inf-sup constant $\Lambda_{s}$ is also independent of the polynomial degree $p$.

Remark 5.7. The above inf-sup condition has been derived under the assumption that (5.16) holds, hence limiting the validity of the present analysis to problems with piecewise linear convection fields $\mathbf{b}$. However, an analogous inf-sup condition still holds for general $\mathbf{b}$, if we replace the test space $S_{\mathcal{T}}^{\mathbf{p}}$ by $W_{\mathcal{T}}^{\mathbf{p}}:=\operatorname{span}(v+$ $\left.\alpha v_{s},\left.\quad v_{s}\right|_{\kappa}=\tau_{\kappa} \Pi_{2}(\mathbf{b} \cdot \nabla v), \quad \kappa \in \mathcal{T}, \quad v \in S_{\mathcal{T}}^{\mathbf{p}}\right)$, endowed with the streamline DGFEM-norm $\|v\|_{\tilde{\mathrm{s}}}^{2}:=\mid\|v\|_{\mathrm{DG}}^{2}+$ $\sum_{\kappa \in \mathcal{T}} \tau_{\kappa}\left\|\boldsymbol{\Pi}_{2}(\mathbf{b} \cdot \nabla v)\right\|_{L_{2}(\kappa)}^{2}$. This approach, though, results in suboptimal, with respect to the polynomial degree $p$, a priori error bounds, $c f$. Remark 5.12 below.

\subsection{Error analysis}

In this section, we derive an a priori error bound for the IP DGFEM (5.2). First, we point out that Galerkin orthogonality does not hold due to the inconsistency of $\tilde{B}(\cdot, \cdot)$. Thereby, we derive the following abstract error bound in the spirit of Strang's second lemma.

Lemma 5.8. Let $u$ be the analytical solution of (2.1), (2.4), and $u_{h}$ be the IP DGFEM solution satisfying (5.2). Assuming the inf-sup condition derived in Theorem 5.5 holds, we have that

$$
\left\|u-u_{h}\left|\left\|_{\mathrm{s}} \leq \mid\right\| u-\tilde{\Pi} u \|_{\mathrm{s}}+\frac{1}{\Lambda_{s}} \sup _{\omega \in S_{\mathcal{T}}^{\mathrm{p}} \backslash\{0\}} \frac{|\tilde{B}(\tilde{\Pi} u-u, \omega)|}{\left|\|\omega \mid\|_{\mathrm{s}}\right.}+\frac{1}{\Lambda_{s}} \sup _{\omega \in S_{\mathcal{T}}^{\mathrm{P}} \backslash\{0\}} \frac{|\tilde{B}(u, \omega)-\tilde{l}(\omega)|}{\left|\|\omega \mid\|_{\mathrm{s}}\right.},\right.\right.
$$

where $\tilde{\Pi}$ is the operator defined in Lemma 4.8 .

Proof. This follows in a standard manner, based on Strang's second lemma.

We now derive the main result of this paper.

Theorem 5.9. Let $\Omega \subset \mathbb{R}^{d}, d=2,3$, be a bounded polyhedral domain, and $\mathcal{T}=\{\kappa\}$ be a subdivision of $\Omega$ consisting of general polytopic elements satisfying Assumptions 4.1, 4.2 and 4.12. Further, let $\mathcal{T}_{\sharp}=\{\mathcal{K}\}$ denote the associated covering of $\Omega$ consisting of shape-regular d-simplices as in Definition 4.4, which satisfies Assumption 4.5. Let $u_{h} \in S_{\mathcal{T}}^{\mathrm{p}}$ be the IP DGFEM approximation to $u \in H^{1}(\Omega)$, the solution of (2.1), (2.4), defined by (5.2) with the discontinuity-penalization parameter given by (5.6), and suppose that $\left.u\right|_{\kappa} \in H^{l_{\kappa}}(\kappa)$, $l_{\kappa}>1+d / 2$, for each $\kappa \in \mathcal{T}$, such that $\left.\mathfrak{E} u\right|_{\mathcal{K}} \in H^{l_{\kappa}}(\mathcal{K})$, where $\mathcal{K} \in \mathcal{T}_{\sharp}$ with $\kappa \subset \mathcal{K}$. Then, the following error bound holds:

$$
\left\|u-u_{h}\right\|_{\mathrm{s}}^{2} \leq C \sum_{\kappa \in \mathcal{T}} \frac{h_{\kappa}^{2 s_{\kappa}}}{p_{\kappa}^{2 l_{\kappa}}}\left(\mathcal{G}_{\kappa}\left(F, C_{m}, p_{\kappa}, \tau_{\kappa}\right)+\mathcal{D}_{\kappa}\left(F, C_{\mathrm{INV}}, C_{m}, p_{\kappa}\right)\right)\|\mathfrak{E} u\|_{H^{l_{\kappa}(\mathcal{K})}}^{2},
$$


where

$$
\begin{aligned}
\mathcal{G}_{\kappa}\left(F, C_{m}, p_{\kappa}, \tau_{\kappa}\right)= & \left\|c_{0}\right\|_{L_{\infty}(\kappa)}^{2}+\gamma_{\kappa}^{2}+\tau_{\kappa}^{-1}+\tau_{\kappa} \beta_{\kappa}^{2} p_{\kappa}^{2} h_{\kappa}^{-2}+\bar{a}_{\kappa} p_{\kappa}^{2} h_{\kappa}^{-2} \\
& +\beta_{\kappa} p_{\kappa} h_{\kappa}^{-d} \sum_{F \subset \partial \kappa} C_{m}\left(p_{\kappa}, \kappa, F\right)|F|+p_{\kappa} h_{\kappa}^{-d} \sum_{F \subset \partial \kappa \cap\left(\Gamma_{\mathrm{int}} \cup \Gamma_{\mathrm{D}}\right)} C_{m}\left(p_{\kappa}, \kappa, F\right) \sigma|F|,
\end{aligned}
$$

and

$$
\begin{aligned}
\mathcal{D}_{\kappa}\left(F, C_{\mathrm{INV}}, C_{m}, p_{\kappa}\right)= & \bar{a}_{\kappa}\left(p_{\kappa}^{3} h_{\kappa}^{-d-2} \sum_{F \subset \partial \kappa \cap\left(\Gamma_{\mathrm{int}} \cup \Gamma_{\mathrm{D}}\right)} C_{m}\left(p_{\kappa}, \kappa, F\right) \sigma^{-1}|F|\right. \\
& \left.+p_{\kappa}^{4}|\kappa|^{-1} h_{\kappa}^{-2} \sum_{F \subset \partial \kappa \cap\left(\Gamma_{\mathrm{int}} \cup \Gamma_{\mathrm{D}}\right)} C_{\mathrm{INV}}\left(p_{\kappa}, \kappa, F\right) \sigma^{-1}|F|\right),
\end{aligned}
$$

with $s_{\kappa}=\min \left\{p_{\kappa}+1, l_{\kappa}\right\}$ and $p_{\kappa} \geq 1$. Here, $\gamma_{\kappa}=\left\|c_{1}\right\|_{L_{\infty}(\kappa)}$, with $c_{1}(x):=(c(x)-\nabla \cdot \mathbf{b}(x)) /\left(c_{0}(x)\right)$, $c_{0}$ as in (2.5), and $\beta_{\kappa}=\|\mathbf{b}\|_{L_{\infty}(\kappa)}$. The positive constant $C$ is independent of the discretization parameters.

Proof. Our starting point is the bound (5.33) given in Lemma 5.8. To bound the first term on the right-hand side of (5.33), we employ the approximation results in Lemma 4.8, together with Assumption 4.2; thereby, we get

$$
\begin{aligned}
\|u-\tilde{\Pi} u\|_{\mathrm{s}}^{2} \leq & C \sum_{\kappa \in \mathcal{T}} \frac{h_{\kappa}^{2 s_{\kappa}}}{p_{\kappa}^{2 l_{\kappa}}}\left(\left\|c_{0}\right\|_{L_{\infty}(\kappa)}^{2}+\tau_{\kappa}\|\mathbf{b}\|_{L_{\infty}(\kappa)}^{2} \frac{h_{\kappa}^{-2}}{p_{\kappa}^{-2}}+\bar{a}_{\kappa} \frac{h_{\kappa}^{-2}}{p_{\kappa}^{-2}}+\|\mathbf{b}\|_{L_{\infty}(\kappa)} \frac{h_{\kappa}^{-d}}{p_{\kappa}^{-1}} \sum_{F \subset \partial \kappa} C_{m}\left(p_{\kappa}, \kappa, F\right)|F|\right. \\
& \left.+\frac{h_{\kappa}^{-d}}{p_{\kappa}^{-1}} \sum_{F \subset \partial \kappa \cap\left(\Gamma_{\mathrm{int}} \cup \Gamma_{\mathrm{D}}\right)} C_{m}\left(p_{\kappa}, \kappa, F\right) \sigma|F|\right)\|\mathfrak{E} u\|_{H^{l_{\kappa}(\mathcal{K})}}^{2} .
\end{aligned}
$$

Next, we define $\eta=u-\tilde{\Pi} u$ and embark on bounding the second term on right-hand side of (5.33). Exploiting element-wise integration by parts, the advection-reaction bilinear form $B_{\mathrm{ar}}(\cdot, \cdot), c f .(3.3)$, can be bounded as follows:

$$
\begin{aligned}
\left|B_{\mathrm{ar}}(\eta, \omega)\right| \leq & \mid \sum_{\kappa \in \mathcal{T}}\left(\int_{\kappa}(c-\nabla \cdot \mathbf{b}) \omega \eta \mathrm{d} x-\int_{\kappa}(\mathbf{b} \cdot \nabla \omega) \eta \mathrm{d} x+\int_{\partial_{-} \varsigma \Gamma}(\mathbf{b} \cdot \mathbf{n})\lfloor\omega\rfloor \eta^{-} \mathrm{d} s\right. \\
& \left.+\int_{\partial_{+} \kappa \cap \Gamma}(\mathbf{b} \cdot \mathbf{n}) \omega^{+} \eta^{+} \mathrm{d} s\right) \mid \\
\leq & \sum_{\kappa \in \mathcal{T}}\left(\left\|c_{0} \omega\right\|_{L_{2}(\kappa)}\left\|c_{1} \eta\right\|_{L_{2}(\kappa)}+\left\|\tau_{\kappa}^{1 / 2} \mathbf{b} \cdot \nabla \omega\right\|_{L_{2}(\kappa)}\left\|\tau_{\kappa}^{-1 / 2} \eta\right\|_{L_{2}(\kappa)}+\left\|\omega^{+}-\omega^{-}\right\|_{\partial_{-} \kappa \backslash \Gamma}\left\|\eta^{-}\right\|_{\partial_{-} \kappa \backslash \Gamma}\right. \\
& \left.+\left\|\omega^{+}\right\|_{\partial_{+} \kappa \cap \Gamma}\left\|\eta^{+}\right\|_{\partial_{+} \kappa \cap \Gamma}\right) \\
\leq & \left(\sum_{\kappa \in \mathcal{T}}\left\|c_{1} \eta\right\|_{L_{2}(\kappa)}^{2}+\sum_{\kappa \in \mathcal{T}} \tau_{\kappa}^{-1}\|\eta\|_{L_{2}(\kappa)}^{2}+2 \sum_{\kappa \in \mathcal{T}}\left\|\eta^{-}\right\|_{\partial_{-} \kappa \backslash \Gamma}^{2}+2 \sum_{\kappa \in \mathcal{T}}\left\|\eta^{+}\right\|_{\partial_{+} \kappa \cap \Gamma}^{2}\right)^{1 / 2} \\
& \times\left(\|\omega\|_{\mathrm{ar}^{2}}^{2}+\sum_{\kappa \in \mathcal{T}} \tau_{\kappa}\|\mathbf{b} \cdot \nabla \omega\|_{L_{2}(\kappa)}^{2}\right)^{1 / 2} \cdot
\end{aligned}
$$


We now derive a bound for $\tilde{B}(\eta, \omega)$ by employing the above result in conjunction with the continuity of $\tilde{B}_{\mathrm{d}}(\cdot, \cdot)$; thereby, we get

$$
\begin{aligned}
|\tilde{B}(\eta, \omega)|= & \left|B_{\mathrm{ar}}(\eta, \omega)+\tilde{B}_{\mathrm{d}}(\eta, \omega)\right| \\
\leq & \left(\sum_{\kappa \in \mathcal{T}}\left\|c_{1} \eta\right\|_{L_{2}(\kappa)}^{2}+\sum_{\kappa \in \mathcal{T}} \tau_{\kappa}^{-1}\|\eta\|_{L_{2}(\kappa)}^{2}+2 \sum_{\kappa \in \mathcal{T}}\left\|\eta^{-}\right\|_{\partial_{-} \varsigma \backslash \Gamma}^{2}\right. \\
& \left.+2 \sum_{\kappa \in \mathcal{T}}\left\|\eta^{+}\right\|_{\partial_{+} \kappa \cap \Gamma}^{2}\right)^{1 / 2}\left(\|\omega \mid\|_{\mathrm{ar}}^{2}+\sum_{\kappa \in \mathcal{T}} \tau_{\kappa}\|\mathbf{b} \cdot \nabla \omega\|_{L_{2}(\kappa)}^{2}\right)^{1 / 2}+C_{\mathrm{d}}^{\mathrm{cont}}\|\| \eta\left\|_{\mathrm{d}}\right\| \mid \omega \|_{\mathrm{d}} \\
\leq & \left(\sum_{\kappa \in \mathcal{T}} \gamma_{\kappa}^{2}\|\eta\|_{L_{2}(\kappa)}^{2}+\sum_{\kappa \in \mathcal{T}} \tau_{\kappa}^{-1}\|\eta\|_{L_{2}(\kappa)}^{2}+2 \sum_{\kappa \in \mathcal{T}}\left\|\eta^{-}\right\|_{\partial_{-} \kappa \backslash \Gamma}^{2}+2\left\|\eta^{+}\right\|_{\partial_{+} \kappa \cap \Gamma}^{2}\right. \\
& \left.+\left(C_{\mathrm{d}}^{\mathrm{cont}}\right)^{2} \sum_{\kappa \in \mathcal{T}}\|\sqrt{a} \nabla \eta\|_{L_{2}(\kappa)}^{2}+\left(C_{\mathrm{d}}^{\mathrm{cont}}\right)^{2} \int_{\Gamma_{\mathrm{int}} \cup \Gamma_{\mathrm{D}}} \sigma|\llbracket \eta \rrbracket|^{2} \mathrm{~d} s\right)^{1 / 2}\|\| \omega \|_{\mathrm{s} .}
\end{aligned}
$$

Hence, by applying the approximation results in Lemma 4.8, we have the following bound:

$$
\begin{aligned}
\sup _{\omega \in S_{\mathcal{T}}^{\mathbf{p}} \backslash\{0\}} \frac{|\tilde{B}(\tilde{\Pi} u-u, \omega)|}{\left|\|\omega \mid\|_{\mathrm{s}}\right.} \leq & C\left(\sum _ { \kappa \in \mathcal { T } } \frac { h _ { \kappa } ^ { 2 s _ { \kappa } } } { p _ { \kappa } ^ { 2 l _ { \kappa } } } \left(\gamma_{\kappa}^{2}+\tau_{\kappa}^{-1}+\bar{a}_{\kappa} \frac{h_{\kappa}^{-2}}{p_{\kappa}^{-2}}+\|\mathbf{b}\|_{L_{\infty}(\kappa)} \frac{h_{\kappa}^{-d}}{p_{\kappa}^{-1}} \sum_{F \subset \partial \kappa} C_{m}\left(p_{\kappa}, \kappa, F\right)|F|\right.\right. \\
& \left.\left.+\frac{h_{\kappa}^{-d}}{p_{\kappa}^{-1}} \sum_{F \subset \partial \kappa \cap\left(\Gamma_{\mathrm{int}} \cup \Gamma_{\mathrm{D}}\right)} C_{m}\left(p_{\kappa}, \kappa, F\right) \sigma|F|\right)\|\mathbb{E} u\|_{H^{l_{\kappa}}(\mathcal{K})}^{2}\right)^{1 / 2}
\end{aligned}
$$

Finally, we consider the residual due to the inconsistent formulation given by the third term in (5.33). From the definition of the original and inconsistent bilinear forms given by (3.4) and (5.1), respectively, we deduce that

$$
\tilde{B}(u, \omega)-\tilde{l}(\omega)=\int_{\Gamma_{\mathrm{int}} \cup \Gamma_{\mathrm{D}}}\left\{\left\{a\left(\nabla_{h} u-\Pi_{2}\left(\nabla_{h} u\right)\right)\right\} \cdot \llbracket \omega \rrbracket \mathrm{d} s,\right.
$$

where $\boldsymbol{\Pi}_{2}$ denotes the vector-valued $L_{2}$-projection onto the finite element space $\left[S_{\mathcal{T}}^{\mathbf{p}-\mathbf{1}}\right]^{d}, c f .[21]$. Employing the Cauchy-Schwarz inequality gives

$$
\sup _{\omega \in S_{\mathcal{T}}^{\mathbf{P}} \backslash\{0\}} \frac{|\tilde{B}(u, \omega)-\tilde{l}(\omega)|}{|||\omega| \|_{\mathrm{s}}} \leq\left(\int_{\Gamma_{\mathrm{int}} \cup \Gamma_{\mathrm{D}}} \sigma^{-1} \mid\left\{\left.\left\{a\left(\nabla_{h} u-\Pi_{2}\left(\nabla_{h} u\right)\right)\right\}\right|^{2} \mathrm{~d} s\right)^{1 / 2} .\right.
$$

Writing $\tilde{\boldsymbol{\Pi}}$ to denote the vector-valued generalization of the $h p$-projection operator $\tilde{\Pi}$ given in Lemma 4.8 , we note that

$$
\begin{aligned}
\int_{\Gamma_{\mathrm{int}} \cup \Gamma_{\mathrm{D}}} & \sigma^{-1} \mid\left\{\left.\left\{a\left(\nabla_{h} u-\Pi_{2}\left(\nabla_{h} u\right)\right)\right\}\right|^{2} \mathrm{~d} s\right. \\
& \leq 2 \int_{\Gamma_{\mathrm{int}} \cup \Gamma_{\mathrm{D}}} \sigma^{-1}\left|\left\{\left\{a\left(\nabla_{h} u-\tilde{\boldsymbol{\Pi}}\left(\nabla_{h} u\right)\right)\right\}\right\}\right|^{2} \mathrm{~d} s+2 \int_{\Gamma_{\mathrm{int}} \cup \Gamma_{\mathrm{D}}} \sigma^{-1} \mid\left\{\left.\left\{a \Pi_{2}\left(\tilde{\boldsymbol{\Pi}}\left(\nabla_{h} u\right)-\nabla_{h} u\right)\right\}\right|^{2} \mathrm{~d} s\right. \\
& \equiv \mathrm{I}+\mathrm{II} .
\end{aligned}
$$

To bound Term I, proceeding as above gives

$$
\mathrm{I} \leq C \sum_{\kappa \in \mathcal{T}} \bar{a}_{\kappa} \frac{h_{\kappa}^{2\left(s_{\kappa}-1\right)}}{p_{\kappa}^{2\left(l_{\kappa}-1\right)}} \frac{h_{\kappa}^{-d}}{p_{\kappa}^{-1}}\left(\sum_{F \subset \partial \kappa \cap\left(\Gamma_{\mathrm{int}} \cup \Gamma_{\mathrm{D}}\right)} C_{m}\left(p_{\kappa}, \kappa, F\right) \sigma^{-1}|F|\right)\|\mathfrak{E} u\|_{H^{l_{\kappa}(\mathcal{K})}}^{2} .
$$


Exploiting the inverse inequality stated in Lemma 4.11, the $L_{2}$-stability of the projector $\Pi_{2}$, and the approximation results stated in Lemma 4.8, we deduce that

$$
\mathrm{II} \leq C \sum_{\kappa \in \mathcal{T}} \bar{a}_{\kappa} \frac{h_{\kappa}^{2\left(s_{\kappa}-1\right)}}{p_{\kappa}^{2\left(l_{\kappa}-1\right)}} \frac{|\kappa|^{-1}}{p_{\kappa}^{-2}}\left(\sum_{F \subset \partial \kappa \cap\left(\Gamma_{\mathrm{int}} \cup \Gamma_{\mathrm{D}}\right)} C_{\mathrm{INV}}\left(p_{\kappa}, \kappa, F\right) \sigma^{-1}|F|\right)\|\mathfrak{E} u\|_{H^{l_{\kappa}}(\mathcal{K})}^{2} .
$$

Hence, the following bound holds:

$$
\begin{aligned}
\sup _{\omega \in S_{\mathcal{T}}^{\mathrm{p}} \backslash\{0\}} \frac{|\tilde{B}(u, \omega)-\tilde{l}(\omega)| \leq}{\|\omega \mid\|_{\mathrm{s}}} & C\left(\sum _ { \kappa \in \mathcal { T } } \overline { a } _ { \kappa } \frac { h _ { \kappa } ^ { 2 s _ { \kappa } } } { p _ { \kappa } ^ { 2 l _ { \kappa } } } \left(\frac{h_{\kappa}^{-d-2}}{p_{\kappa}^{-3}} \sum_{F \subset \partial \kappa \cap\left(\Gamma_{\mathrm{int}} \cup \Gamma_{\mathrm{D}}\right)} C_{m}\left(p_{\kappa}, \kappa, F\right) \sigma^{-1}|F|\right.\right. \\
& \left.\left.+\frac{|\kappa|^{-1} h_{\kappa}^{-2}}{p_{\kappa}^{-4}} \sum_{F \subset \partial \kappa \cap\left(\Gamma_{\mathrm{int}} \cup \Gamma_{\mathrm{D}}\right)} C_{\mathrm{INV}}\left(p_{\kappa}, \kappa, F\right) \sigma^{-1}|F|\right)\|\mathfrak{E} u\|_{H^{l_{\kappa}}(\mathcal{K})}^{2}\right)^{1 / 2} .
\end{aligned}
$$

Finally, the error bound (5.34) follows by combining the error bounds in (5.37), (5.38), (5.39) with Lemma 5.8.

Remark 5.10. We note that the above $h p$-version a priori bound for the IP DGFEM (5.2) holds without the need to impose any assumption concerning the relative size of the faces $F, F \subset \partial \kappa$, of a given polytopic element $\kappa \in \mathcal{T}$. If $\mathbf{b} \equiv \mathbf{0}$ and $c \equiv 0$ on $\Omega$, then the streamline DGFEM-norm degenerates to the diffusion DGFEM-norm $\|\cdot \mid\|_{\mathrm{d}}$ defined in (5.4) which is independent of $\tau_{\kappa}$ with constants $\beta_{\kappa}$ and $\gamma_{\kappa}$ equal to zero and the underlying PDE becomes the pure diffusion problem. Furthermore, the inf-sup condition is equivalent to the coercivity of the bilinear form $\tilde{B}_{\mathrm{d}}(\cdot, \cdot)$. This can be used to derive an error bound, analogous to the error bound (5.34), which generalises the result presented in [21] for the Poisson equation with constant diffusion. Moreover, in this setting, for uniform orders $p_{\kappa}=p \geq 1, h=\max _{\kappa \in \mathcal{T}} h_{\kappa}, s_{\kappa}=s, s=\min \{p+1, l\}, l>1+d / 2$, under the assumption that the diameter of the faces of each element $\kappa \in \mathcal{T}$ is of comparable size to the diameter of the corresponding element, i.e., $\operatorname{diam}(F) \sim h_{\kappa}, h_{\kappa}^{\perp} \sim h_{\kappa}, F \subset \partial \kappa, \kappa \in \mathcal{T}$, so that $|F| \sim h_{\kappa}^{(d-1)}$, the a priori error bound of Theorem 5.9 reduces to

$$
\left\|u-u_{h}\right\|_{\mathrm{d}} \leq C \frac{h^{s-1}}{p^{l-\frac{3}{2}}}\|u\|_{H^{l}(\Omega)} .
$$

This coincides with the analogous result derived in [36] for standard meshes consisting of simplices or tensorproduct elements. Here, we have employed Lemma 4.8 and Theorem 4.7, together with Assumption 4.5, assuming that for such element domains $C_{\mathrm{INV}}\left(p_{\kappa}, F\right)=\mathcal{O}(1)$ and $C_{m}\left(p_{\kappa}, F\right)=\mathcal{O}(1)$ uniformly for each face $F \subset \partial \kappa$ for all $\kappa \in \mathcal{T}$. This error bound is $h$ optimal and $p$ suboptimal by $p^{1 / 2}$.

Remark 5.11. Consider the purely hyperbolic case when the diffusion tensor $a \equiv 0$. In this case, the constants $\bar{a}_{\kappa}$ and $\tilde{\sigma}_{\kappa}$ are identically zero and the term $\mathcal{D}_{\kappa}\left(F, C_{\mathrm{INV}}, C_{m}, p_{\kappa}\right)$ vanishes due to the consistency of the bilinear form $B_{\mathrm{ar}}(\cdot, \cdot)$. Then, the streamline DGFEM-norm is actually stronger than the advection-reaction DGFEM-norm $\|\cdot \mid\|_{\text {ar }}$ defined in (5.3) and $\tau_{\kappa}=\mathcal{O}\left(\frac{h_{\kappa}}{p_{\kappa}^{2}}\right)$ by (5.10). In this case, for uniform orders, $c f$. Remark 5.10 above, the a priori error bound of Theorem 5.9 yields

$$
\left\|u-u_{h}\left|\left\|_{\text {ar }} \leq \mid\right\| u-u_{h}\left\|_{\mathrm{s}} \leq C \frac{h^{s-\frac{1}{2}}}{p^{l-1}}\right\| u \|_{H^{l}(\Omega)} .\right.\right.
$$

Hence, the above $h p$-bound is optimal in $h$ and suboptimal in $p$ by $p^{1 / 2}$. In this case, our bound generalizes the error estimate derived in [36] to general polytopic meshes under the same assumption $\mathbf{b} \cdot \nabla \xi \in S_{\mathcal{T}}^{\mathbf{p}}, \xi \in S_{\mathcal{T}}^{\mathbf{p}}$.

Remark 5.12. As noted in Remark 5.7, the case of general convection fields $\mathbf{b}$ can be treated, based on employing an inf-sup condition with different test and trial spaces. In this setting, the present analysis can 
easily be adapted to utilize such an inf-sup condition, together with the exploitation of the $L_{2}$-projector $\Pi_{2}$ onto the polytopic element $\kappa \in \mathcal{T}$; however, this yields an error bound in the $\|\cdot \mid\|_{\text {ar }}$ norm that is optimal in $h$ but suboptimal in $p$ by $p^{3 / 2}$ for the purely hyperbolic problem. We also point out that if we modify the DGFEM by including the streamline-diffusion stabilization term as in [34], then an $h p$-optimal bound can be derived without the assumption that $\mathbf{b} \cdot \nabla_{h} \xi \in S_{\mathcal{T}}^{\mathbf{p}}, \xi \in S_{\mathcal{T}}^{\mathbf{p}}$.

\section{NumericAl EXAMPLES}

We present a series of computational examples to numerically investigate the asymptotic convergence behaviour of the proposed IP DGFEM on general meshes consisting of polytopic elements. As in [21], the integrals arising in the bilinear and linear forms $B(\cdot, \cdot)$ and $\ell(\cdot)$, respectively, are computed based on employing a quadrature scheme defined on a sub-tessalation of each polytopic element in the underlying finite element mesh. Throughout this section, the IP DGFEM solution $u_{h}$ defined by (3.2) is computed with the constant $C_{\sigma}$ appearing in the discontinuity-penalization parameter $\sigma$ defined in Lemma 5.1 equal to 10. Given the computations already presented in [21] for pure diffusion problems, here we concentrate on studying the performance of the proposed IP DGFEM in the hyperbolic and mixed parabolic-hyperbolic setting. To this end, we first study a pure hyperbolic problem (diffusion tensor $a \equiv 0$ ) in Section 6.1. Secondly, we consider an advectiondiffusion-reaction problem with degenerate, anisotropic diffusion tensor $a$ in Section 6.2. Within these examples, we employ polygonal meshes generated using the general-purpose mesh generator PolyMesher, cf. [48]. Finally, in Section 6.3, we study the convergence behaviour of the underlying DGFEM for a purely hyperbolic problem in three dimensions on general polytopes generated based on employing agglomeration.

Throughout this section, we compare the performance of employing $\mathcal{P}_{p}$-polynomial bases on polytopic meshes, with $\mathcal{P}_{p^{-}}$and $\mathcal{Q}_{p}$-polynomial bases defined on standard tensor-product meshes.

\subsection{Example 1}

In this first example, we let $\Omega$ be the square domain $(-1,1)^{2}$, and choose

$$
a \equiv 0, \quad \mathbf{b}=\left(2-y^{2}, 2-x\right), \quad c=1+(1+x)(1+y)^{2} ;
$$

the forcing function $f$ is selected so that the analytical solution to $(2.1),(2.4)$ is given by

$$
u(x, y)=1+\sin \left(\pi(1+x)(1+y)^{2} / 8\right)
$$

cf. [36].

We investigate the asymptotic behaviour of the $h p$-version DGFEM on a sequence of successively finer polygonal and uniform quadrilateral meshes for different values of the polynomial degree $p$. Three settings are compared: uniform quadrilateral meshes and local polynomial bases consisting of either $\mathcal{P}_{p}$ or $\mathcal{Q}_{p}$ polynomials, and polygonal meshes and local polynomial bases consisting of $\mathcal{P}_{p}$ polynomials; the three cases are referred to as, respectively, DGFEM(P), DGFEM(Q), and DGFEM. The polygonal meshes used for DGFEM are generated using the Polymesher mesh generator, $c f$. [48]; a typical mesh, consisting of 256 elements, is depicted in Figure 1.

We first examine the convergence behaviour of the three schemes with respect to $h$-refinement, with fixed polynomial $p$, for $p=1, \ldots, 6$. In Figure 2 we plot the error, measured in terms of both the $L_{2}(\Omega)$ and DGFEMnorm, against the square root of the number of degrees of freedom in the underlying finite element space $S_{\mathcal{T}}^{\mathrm{p}}$. Here, we clearly observe that $\left\|u-u_{h}\right\|_{L_{2}(\Omega)}$ and \|\|$u-u_{h} \mid \|_{\text {DG }}$ converge to zero at the optimal rates $\mathcal{O}\left(h^{p+1}\right)$ and $\mathcal{O}\left(h^{p+\frac{1}{2}}\right)$, respectively, as the mesh size $h$ tends to zero for each fixed $p$. The latter set of results confirm the optimality of Theorem 5.9, cf. Remark 5.11, in the case when polygonal elements are employed. We point out that the (optimal) convergence rate observed when the error is measured in terms of the $L_{2}(\Omega)$ norm is not guaranteed on general meshes, $c f$. [44] (optimal convergence of $\left\|u-u_{h}\right\|_{L_{2}(\Omega)}$ has been established in $[26,27]$, but only for special classes of triangular elements.) From Figure 2, we also observe that polygonal and square meshes deliver almost identical results given the same number of degrees of freedom, when $\mathcal{P}_{p}$ elements are used 


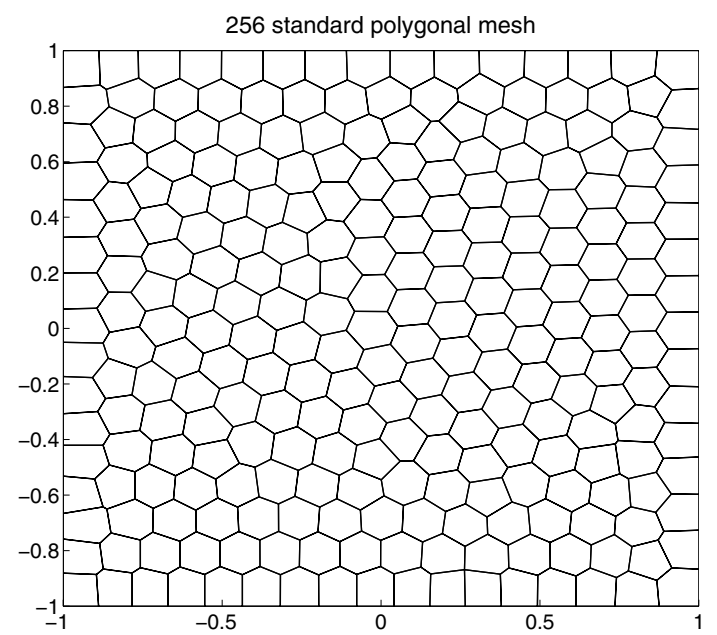

Figure 1. Example 1: Uniform polygonal mesh, consisting of 256 elements.
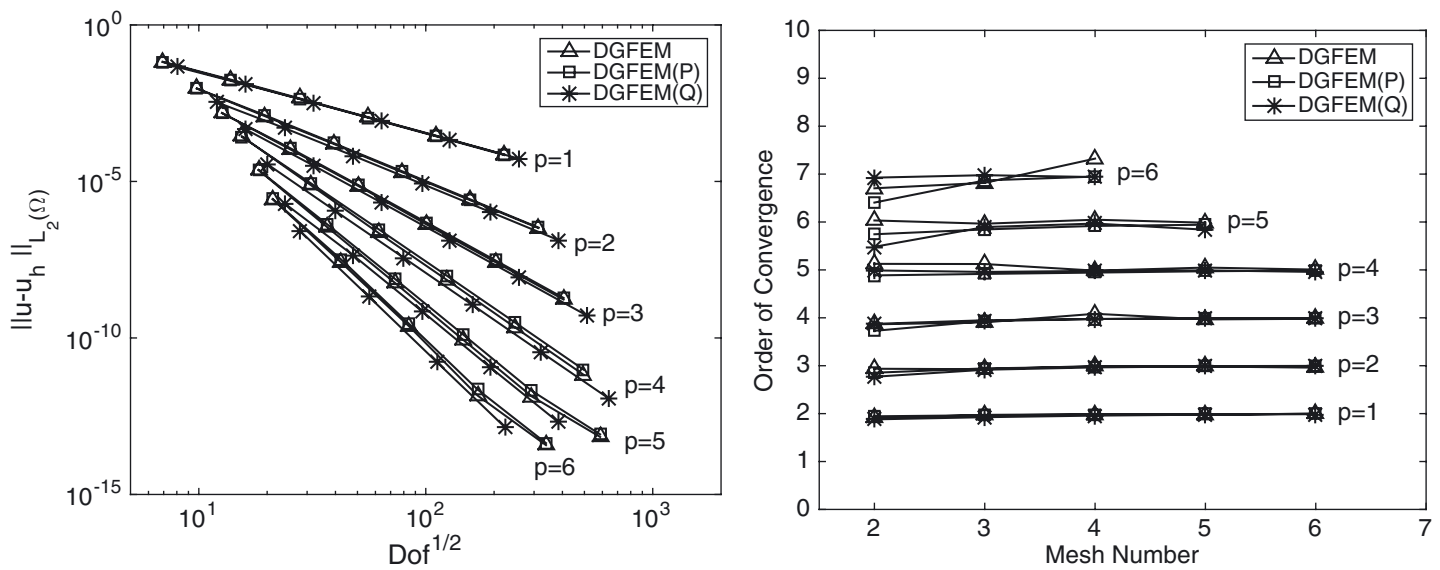

(a)
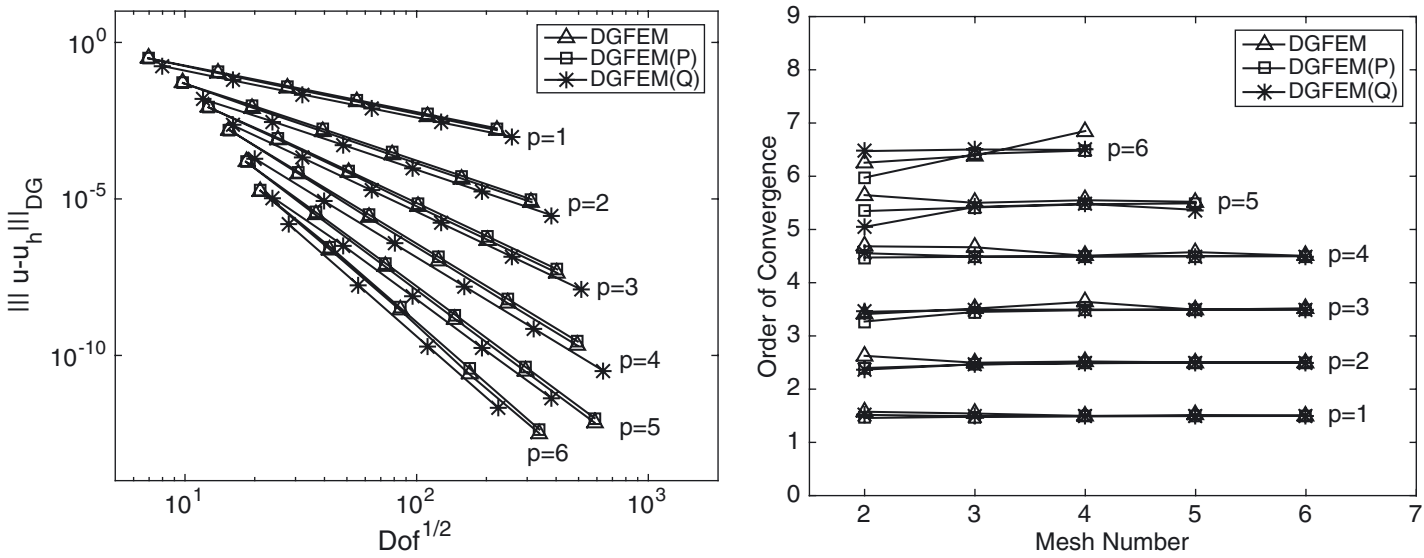

(b)

Figure 2. Example 1: Convergence of the DGFEM under $h$-refinement for $p=1,2, \ldots, 6$. (a) $\left\|u-u_{h}\right\|_{L_{2}(\Omega)}$; (b) $\mid\left\|u-u_{h}\right\|_{\mathrm{DG}}$. 

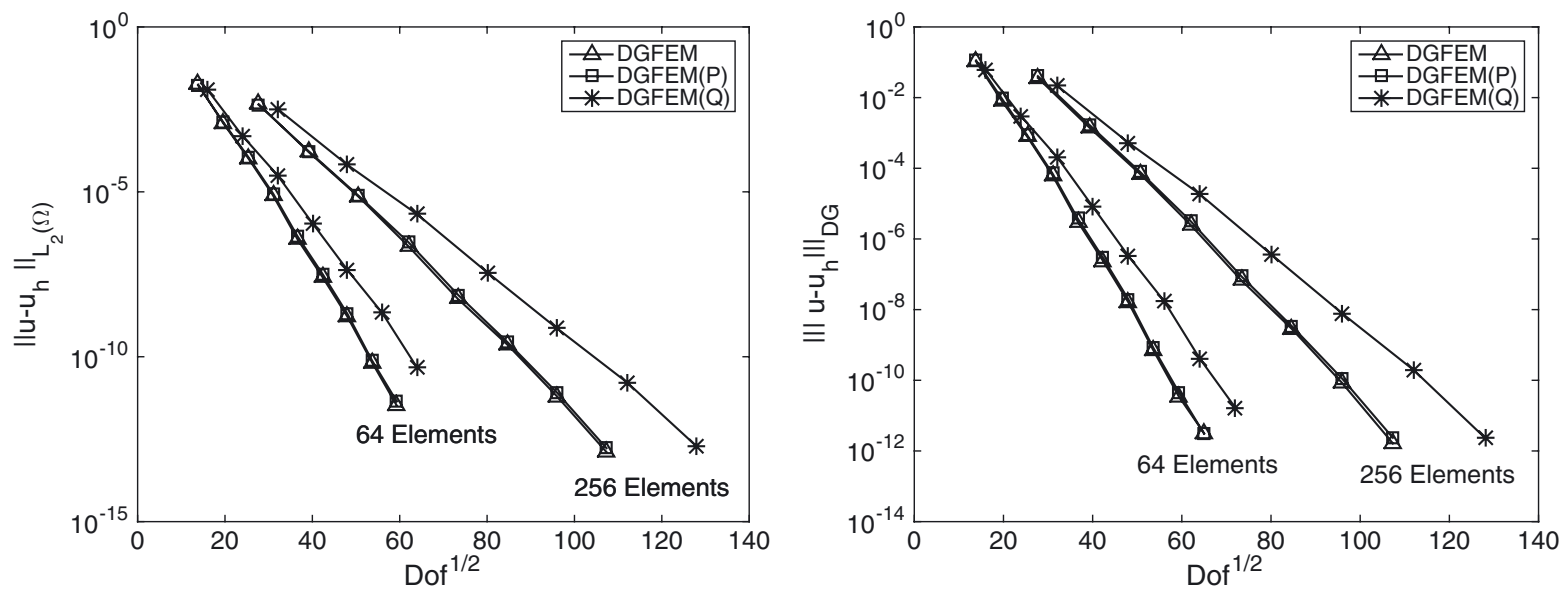

(a)
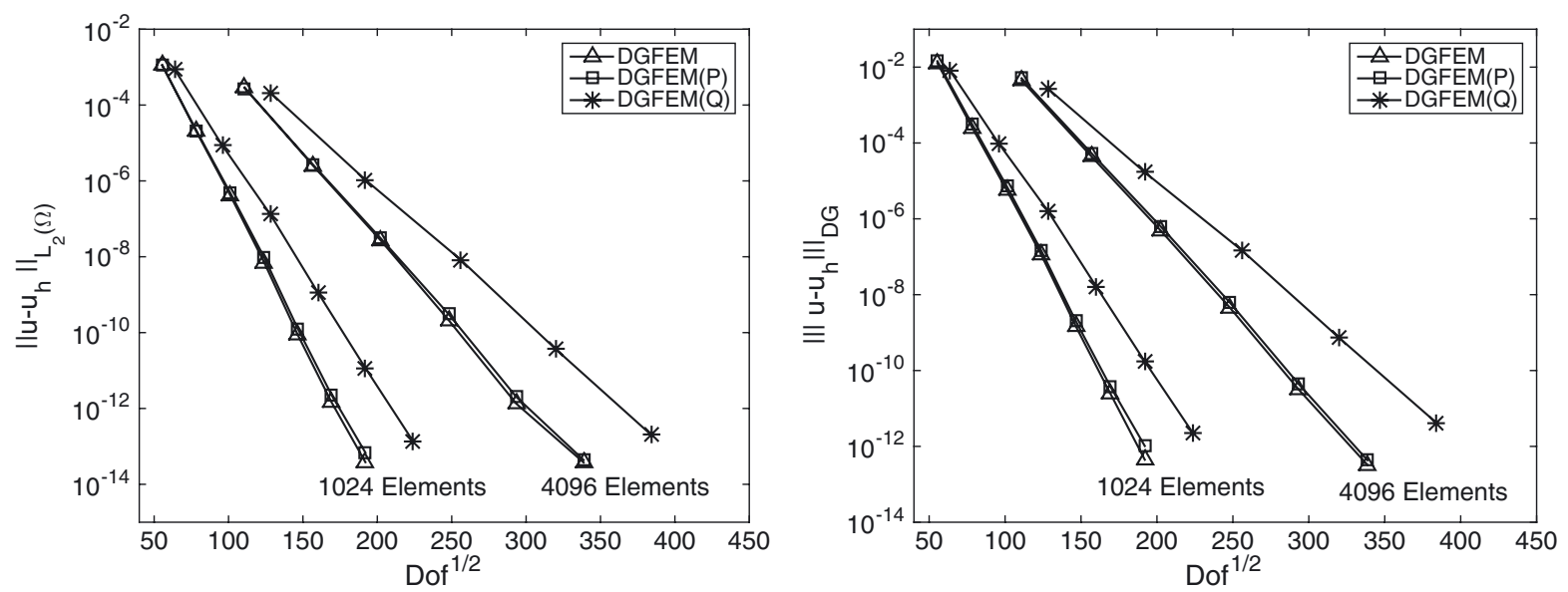

(b)

Figure 3. Example 1: convergence of the DGFEM under p-refinement. Left: $\left\|u-u_{h}\right\|_{L_{2}(\Omega)}$; Right: $\left|\left\|u-u_{h} \mid\right\|_{\mathrm{DG}}\right.$; (a) meshes consisting of 64 and 256 elements; (b) meshes consisting of 1024 and 4096 elements.

(cf. the errors attained by DGFEM and DGFEM(P)). By comparison, the use of tensor-product polynomials, i.e. the $\operatorname{DGFEM}(\mathrm{Q})$ scheme, leads to a marginal decrease in both error quantities.

Finally, in Figure 3 we investigate the convergence behaviour of the three schemes under $p$-refinement, for fixed $h$. Here, uniform polygonal and square meshes consisting of 64, 256, 1024, and 4096 elements are employed. For each mesh, we plot $\left\|u-u_{h}\right\|_{L_{2}(\Omega)}$ and \|\|$u-u_{h} \|_{\text {DG }}$ against the square root of the number of degrees of freedom in $S_{\mathcal{T}}^{\mathrm{p}}$. In each case we clearly observe exponential convergence. We observe that, under $p$-refinement, the efficiency of employing local $\mathcal{P}_{p}$ polynomials is apparent. Indeed, both the DGFEM and DGFEM(P) schemes lead to a significant reduction in the error, when measured in terms of both the $L_{2}(\Omega)$ and DGFEM-norms, for a fixed number of degrees of freedom, when compared with the DGFEM(Q) scheme, cf. [21]. As before, the DGFEM and DGFEM(P) schemes give almost identical results in terms of the size of the discretization error, for a fixed number of degrees of freedom, though in some instances, the former scheme is slightly more accurate. 


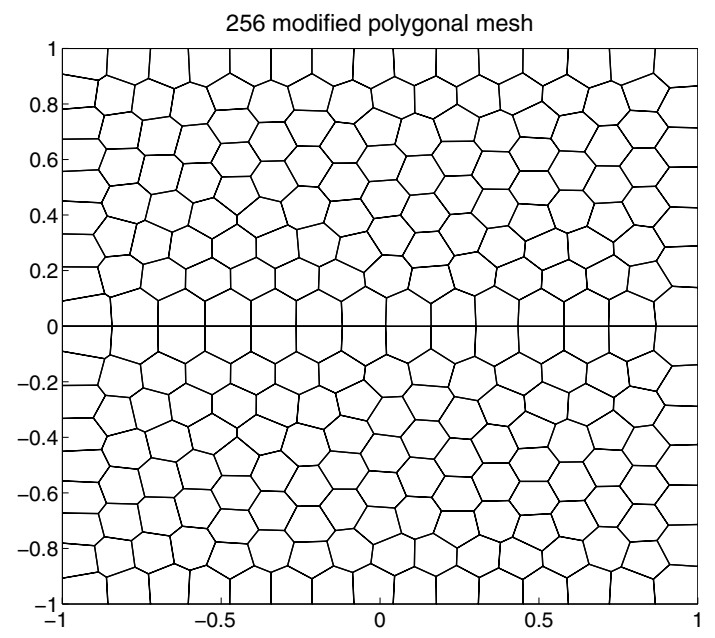

Figure 4. Example 2: Modified uniform polygonal mesh, consisting of 256 elements.

\subsection{Example 2}

In this second example, we consider a partial differential equation with nonnegative characteristic form of mixed type. To this end, we let $\Omega=(-1,1)^{2}$, and consider the PDE problem:

$$
\begin{cases}-x^{2} u_{y y}+u_{x}+u=0, & \text { for }-1 \leq x \leq 1, y>0 \\ u_{x}+u=0, & \text { for }-1 \leq x \leq 1, y \leq 0\end{cases}
$$

with analytical solution:

$$
u(x, y)= \begin{cases}\sin \left(\frac{1}{2} \pi(1+y)\right) \exp \left(-\left(x+\frac{\pi^{2} x^{3}}{12}\right)\right), & \text { for }-1 \leq x \leq 1, y>0 \\ \sin \left(\frac{1}{2} \pi(1+y)\right) \exp (-x), & \text { for }-1 \leq x \leq 1, y \leq 0\end{cases}
$$

$c f$. [30]. This problem is hyperbolic in the region $y \leq 0$ and parabolic for $y>0$. In order to ensure continuity of the normal flux across $y=0$, where the partial differential equation changes type, the analytical solution has a discontinuity across the line $y=0, c f$. [36].

To highlight one of the advantages of employing finite element methods with discontinuous piecewise polynomial spaces, we consider a special class of quadrilateral and polygonal meshes for which the discontinuity in the analytical solution lies on element interfaces only; for the case when polygonal elements are employed, a typical mesh is shown in Figure 4. In this setting, following [36], we modify the discontinuity-penalization parameter $\sigma, c f$. (5.6), so that $\sigma$ vanishes on edges which form part of the interface $y=0$; this ensures that the (physical) discontinuity present in the analytical solution is not penalized within the underlying scheme. In this case, the $h p$-DGFEM behaves as if the analytical solution were smooth, in the sense that exponential rates of convergence are observed for both the $L_{2}(\Omega)$ and DGFEM-norm of the error under p-refinement, $c f$. Figure 5. As in the previous example, we again observe that the slope of the convergence curves for both the DGFEM and DGFEM(P) schemes are steeper than the corresponding convergence curve obtained when local polynomial bases consisting of tensor-product polynomials ( $\mathcal{Q}_{p}$ basis) are employed, $c f$. the numerical results presented for the DGFEM(Q) scheme. The DGFEM and DGFEM(P) schemes give once more very similar results in terms of the size of the computed error for a given number of degrees of freedom. Nevertheless, we notice more clearly that the use of polygonal elements leads to a slight improvement when considering $\left\|u-u_{h}\right\|_{L_{2}(\Omega)}$. As noted in [21], $c f$. also [36], the improvement in the $L_{2}(\Omega)$ norm when polygons are employed, in comparison with square elements, is attributed to the increase in interelement communication. 

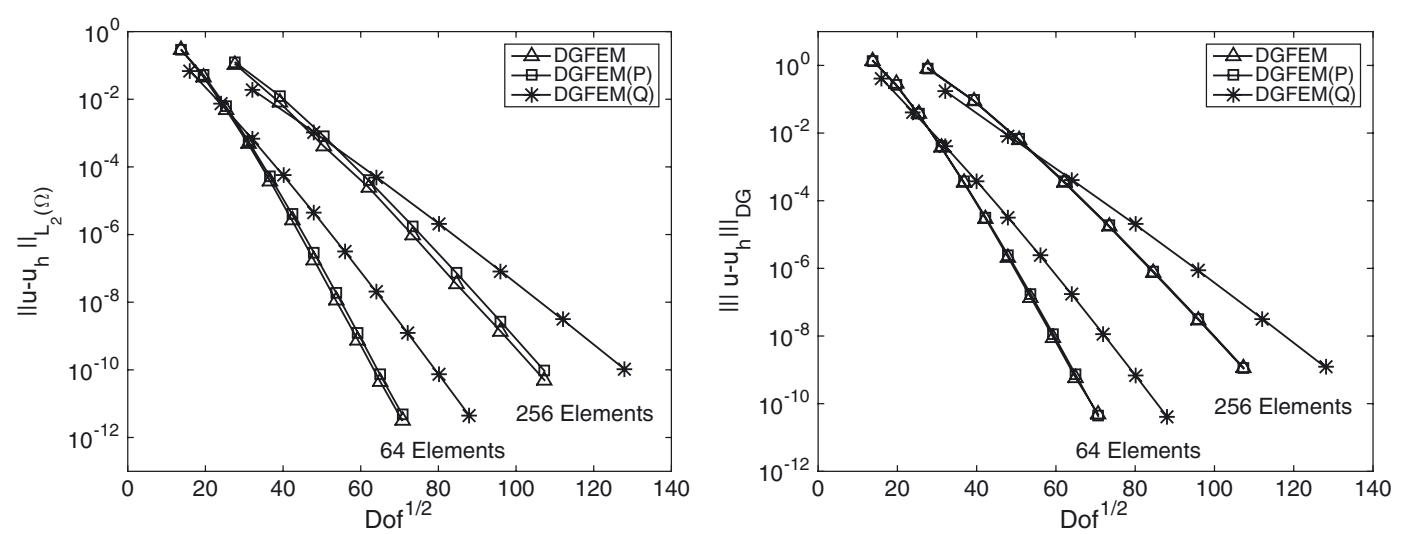

(a)
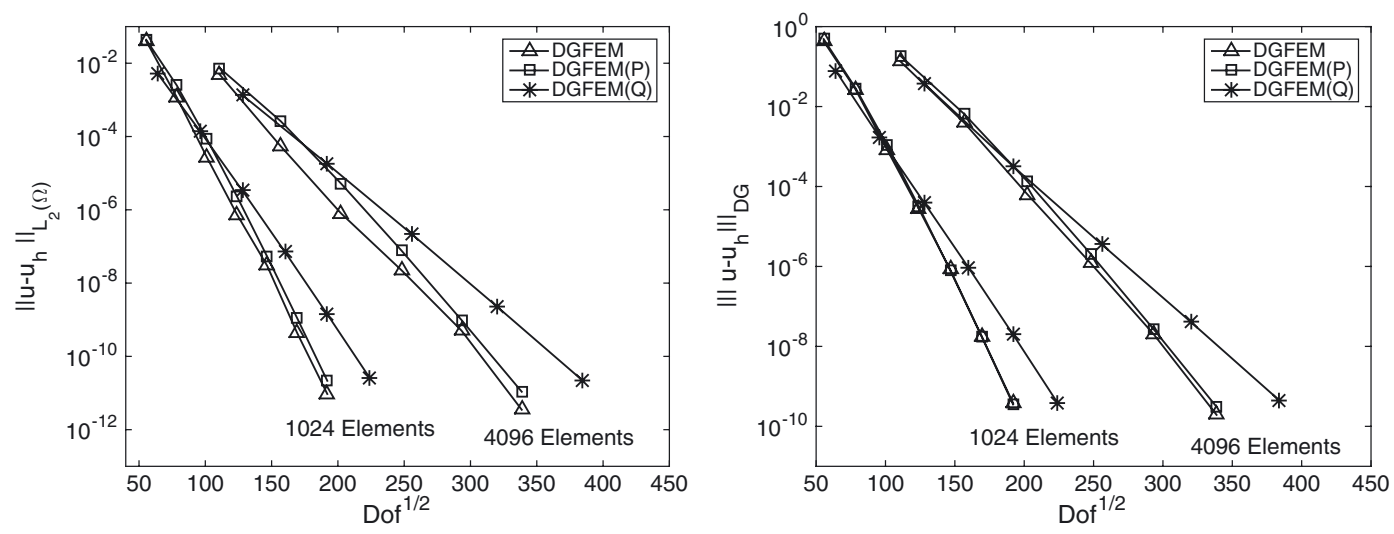

(b)

Figure 5. Example 2: convergence of the DGFEM under p-refinement. Left: $\left\|u-u_{h}\right\|_{L_{2}(\Omega)}$; Right: ||$u-u_{h} \|_{\mathrm{DG}}$; (a) meshes consisting of 64 and 256 elements; (b) meshes consisting of 1024 and 4096 elements.

\subsection{Example 3}

In this final example, we investigate the performance of the proposed DGFEM on sequences of polyhedral meshes in three dimensions for a purely hyperbolic problem. To this end, we consider a three-dimensional variant of the two-dimensional problem considered in Section 6.1. In particular, we let $\Omega$ be the unit cube $(0,1)^{3}$ and set

$$
a \equiv 0, \quad \mathbf{b}=(-y, z, x), \quad c=x y^{2} z ;
$$

$f$ is then selected so that the analytical solution to $(2.1),(2.4)$ is

$$
u(x, y)=1+\sin \left(\pi x y^{2} z / 8\right) .
$$

In this section the DGFEM solution is computed on general polyhedral meshes, stemming from the agglomeration of a given (fixed) fine mesh $\mathcal{I}_{f}$. More precisely, we employ a fine mesh consisting of approximately $1 \mathrm{M}$ tetrahedral elements (1019674 elements, to be precise). The coarse agglomerated mesh $\mathcal{T}$ is then constructed based on exploiting the graph partitioning package METIS [38]. In order for METIS to partition the mesh $\mathcal{T}_{f}$, the logical structure of the mesh is first stored in the form of a graph, where each node represents an element domain of $\mathcal{T}_{f}$, and each link between two nodes represents a face shared by the two elements represented by 


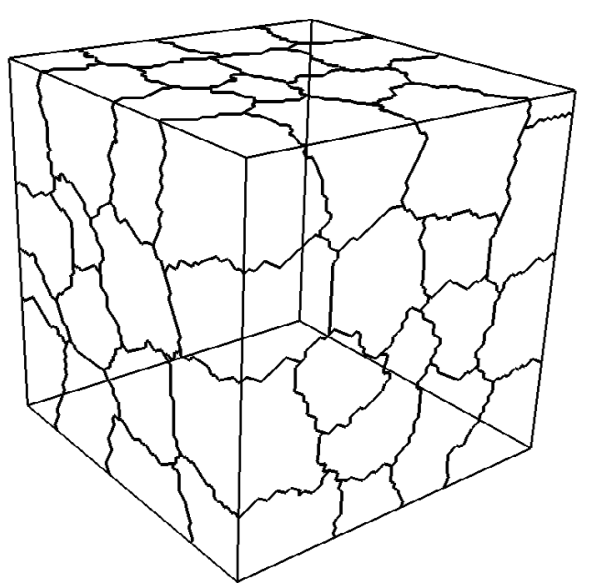

(a)

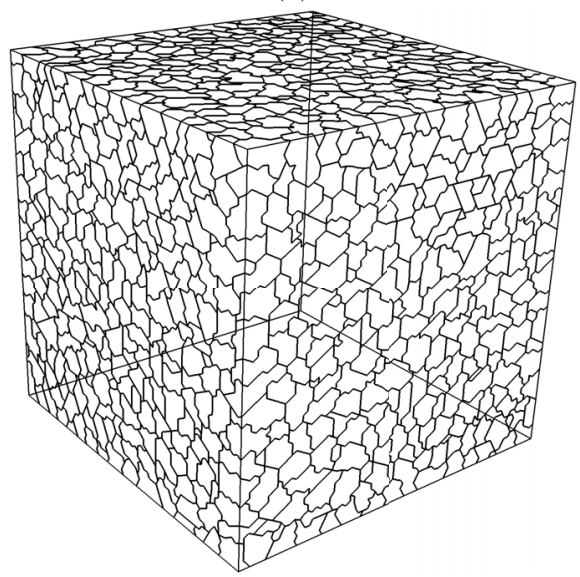

(c)

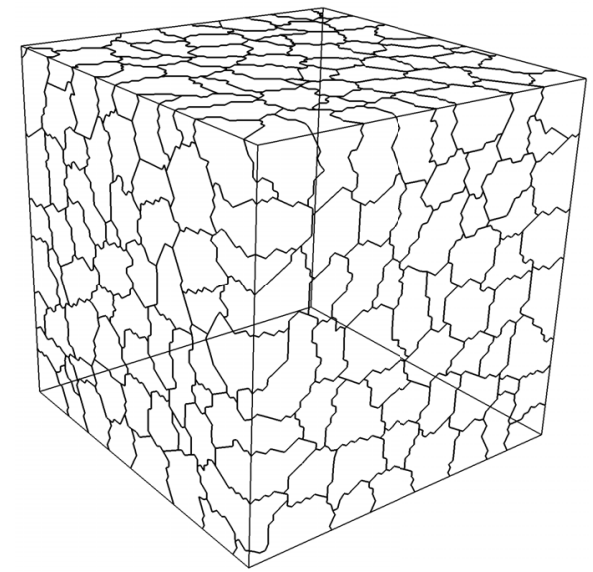

(b)

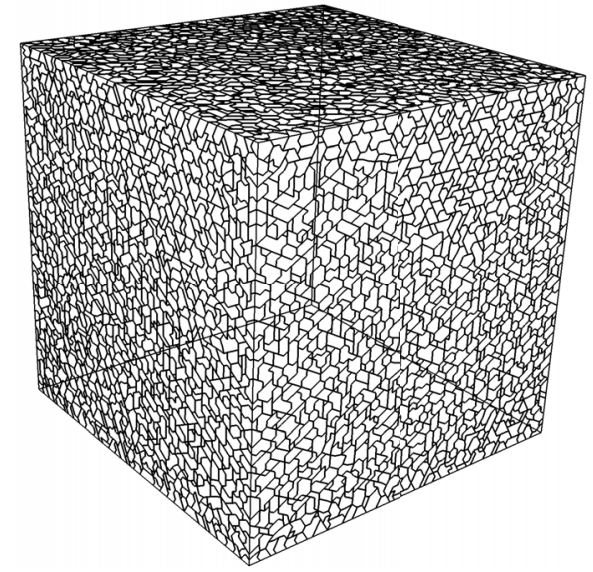

(d)

Figure 6. Example 3: agglomerated meshes. (a) 64 elements; (b) 512 elements; (c) 4096 elements; (d) 32768 elements.

the graph nodes. The partition of $\mathcal{T}_{f}$ constructed by METIS is produced with the objective of minimizing the number of neighbours among each of the resulting partitions. In Figure 6, we show (the surface mesh of) the polyhedral meshes generated by METIS, which consist of 64, 512, 4096, and 32768 elements.

In Figure 7 we investigate the $h$-version convergence behaviour of the DGFEM on both the polyhedral meshes depicted in Figure 6 and uniform hexahedral meshes, using local $\mathcal{P}_{p}$ polynomial bases; denoted by DGFEM and $\operatorname{DGFEM}(\mathrm{P})$, respectively. As already noted in Section 6.1 , we again observe that $\left\|u-u_{h}\right\|_{L_{2}(\Omega)}$ and $\left\|u-u_{h} \mid\right\|_{\mathrm{DG}}$ converge to zero at the optimal rates $\mathcal{O}\left(h^{p+1}\right)$ and $\mathcal{O}\left(h^{p+\frac{1}{2}}\right)$, respectively, as the mesh size $h$ tends to zero for each fixed $p$ when the $\operatorname{DGFEM}(\mathrm{P})$ scheme is employed on uniform tensor-product elements. Moreover, we observe that the DGFEM-norm of the error, when general polyhedral elements are employed, is very similar to the corresponding quantity computed for the $\operatorname{DGFEM}(\mathrm{P})$ scheme. However, we observe a slight degradation of $\left\|u-u_{h}\right\|_{L_{2}(\Omega)}$, when the DGFEM scheme is employed, when compared to the case when uniform hexahedral elements are exploited. For brevity, the corresponding results for the DGFEM(Q) are omitted; though, we note again that, for fixed $p$, this approach is more efficient as the mesh is uniformly refined.

Finally, we study the performance of the DGFEM, DGFEM(P), and DGFEM(Q) schemes under p-refinement, for a given fixed mesh. To this end, in Figure 8 we plot both $\left\|u-u_{h}\right\|_{L_{2}(\Omega)}$ and $\left\|u-u_{h}\right\|_{\text {DG }}$ against the third 

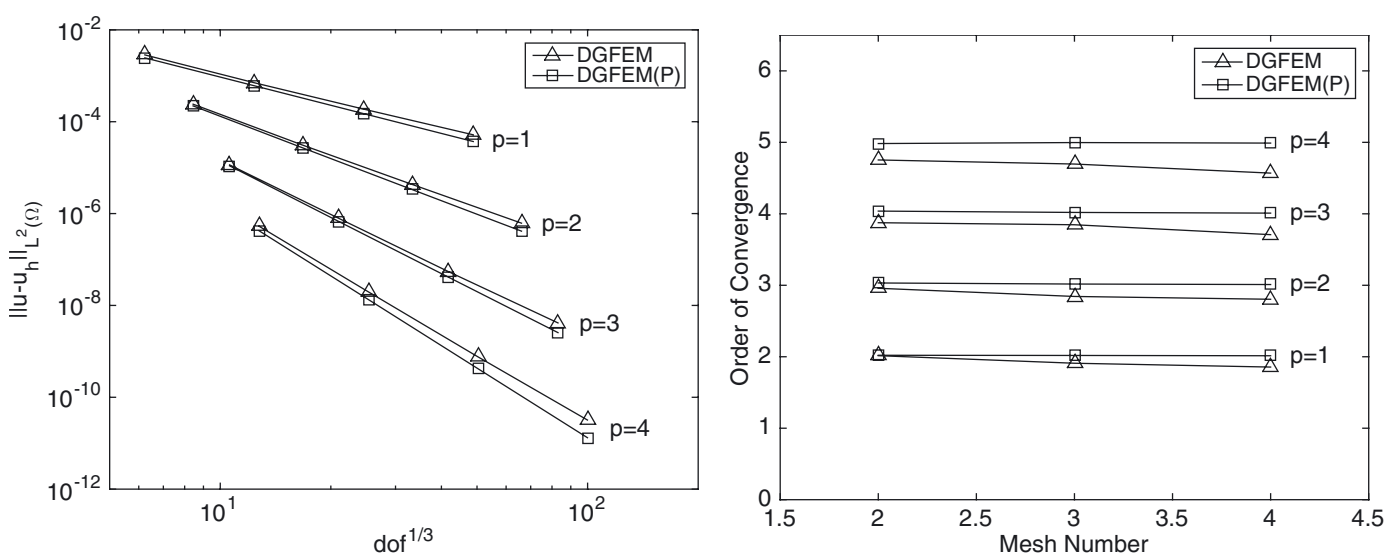

(a)
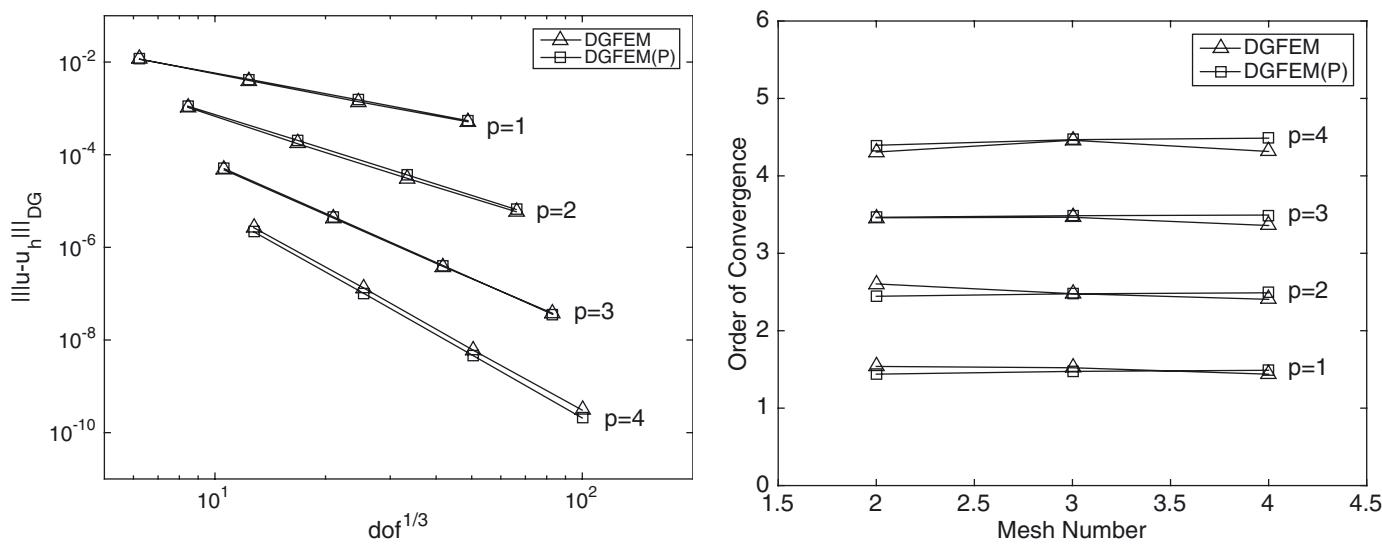

(b)

Figure 7. Example 3: convergence of the DGFEM under $h$-refinement for $p=1,2,3,4$. (a) $\left\|u-u_{h}\right\|_{L_{2}(\Omega)} ;(\mathrm{b}) \mid\left\|u-u_{h}\right\|_{\mathrm{DG}}$.

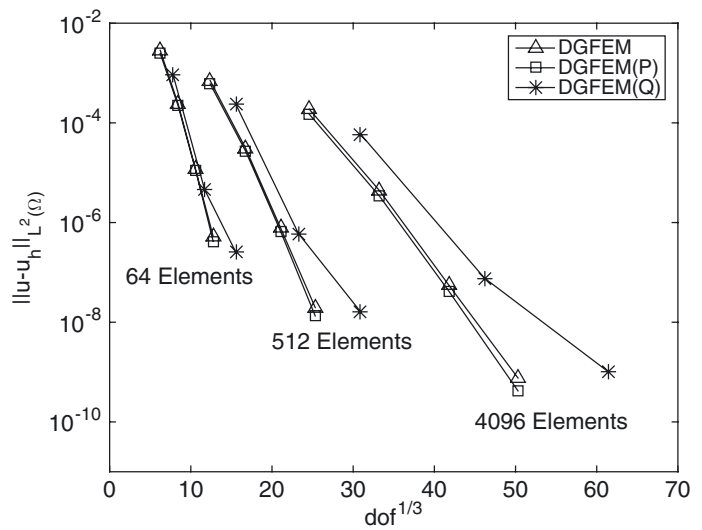

(a)

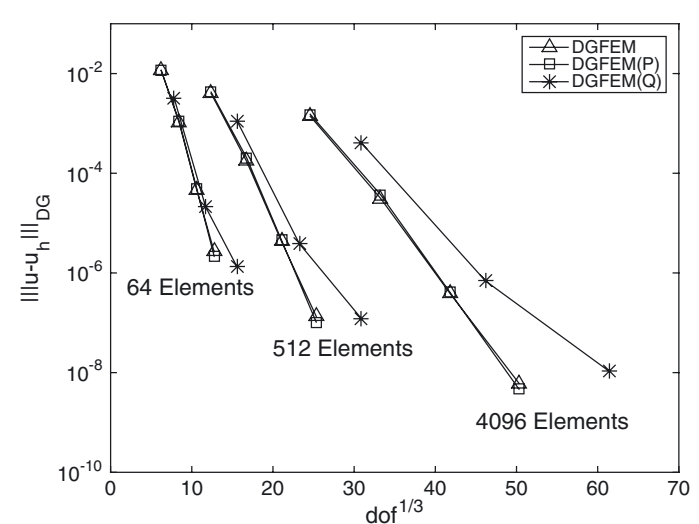

(b)

Figure 8. Example 3: convergence of the DGFEM under $p$-refinement. (a) $\left\|u-u_{h}\right\|_{L_{2}(\Omega)}$; (b) $\left\|u-u_{h}\right\|_{\mathrm{DG}}$. 
root of the number of degrees of freedom in $S_{\mathcal{T}}^{\mathrm{p}}$. As in the previous numerical examples, we again observe the superiority of employing local polynomial bases of total degree $p$ in comparison with full tensor-product bases of degree $p$ in each coordinate direction.

\section{CONCluding REMARKS}

In this article, we have analyzed the $h p$-version of the DGFEM for PDEs with nonnegative characteristic form on general meshes, consisting of polytopic elements. Here, general classes of polytopic elements are admitted, including elements with degenerating $(d-k)$-dimensional facets, $k=1, \ldots, d-1$. The underlying analysis exploits novel $h p$-version approximation and inverse inequalities, together with the inf-sup condition derived in Theorem 5.5; this latter result generalizes the corresponding condition developed in [19,20]. Numerical experiments have been presented which not only confirm the theoretical results derived in this paper, but also demonstrate the efficiency of employing local polynomial spaces of total degree $p$, defined in the physical coordinate system, compared with tensor-product polynomial bases, mapped from a given reference or canonical frame, under $p$-refinement.

Acknowledgements. AC was partially supported by the EPSRC (Grant EP/L022745/1).

\section{REFERENCES}

[1] P.F. Antonietti and B. Ayuso, Schwarz domain decomposition preconditioners for discontinuous Galerkin approximations of elliptic problems: non-overlapping case. M2AN 41 (2007) 21-54.

[2] P.F. Antonietti and B. Ayuso, Multiplicative Schwarz methods for discontinuous Galerkin approximations of elliptic problems. M2AN 42 (2008) 443-469.

[3] P.F. Antonietti and P. Houston, A class of domain decomposition preconditioners for $h p$-discontinuous Galerkin finite element methods. J. Sci. Comput. 46 (2011) 124-149.

[4] P.F. Antonietti, S. Giani and P. Houston, $h p$-Version composite discontinuous Galerkin methods for elliptic problems on complicated domains. SIAM J. Sci. Comput. 35 (2013) A1417-A1439.

[5] P.F. Antonietti, S. Giani and P. Houston, Domain decomposition preconditioners for Discontinuous Galerkin methods for elliptic problems on complicated domains. J. Sci. Comput. 60 (2014) 203-227.

[6] P.F. Antonietti, P. Houston, M. Sarti and M. Verani, Multigrid algorithms for $h p$-version interior penalty discontinuous Galerkin methods on polygonal and polyhedral meshes. Preprint arXiv:1412.0913 (2014).

[7] P.F. Antonietti, M. Sarti and M. Verani, Multigrid algorithms for $h p$-Discontinuous Galerkin discretizations of elliptic problems. SIAM J. Numer. Anal. 53 (2015) 598-618.

[8] D.N. Arnold, F. Brezzi, B. Cockburn and L.D. Marini, Unified analysis of discontinuous Galerkin methods for elliptic problems. SIAM J. Numer. Anal. 39 (2001) 1749-1779.

[9] B. Ayuso and L.D. Marini, Discontinuous Galerkin methods for advection-diffusion-reaction problems. SIAM J. Numer. Anal. 47 (2009) 1391-1420.

[10] I. Babuška, The finite element method with penalty. Math. Comput. 27 (1973) 221-228.

[11] I. Babuška and M. Suri, The $h-p$ version of the finite element method with quasi-uniform meshes. RAIRO Modél. Math. Anal. Numér. 21 (1987) 199-238.

[12] I. Babuška and M. Suri, The optimal convergence rate of the p-version of the finite element method. SIAM J. Numer. Anal. 24 (1987) 750-776.

[13] G.A. Baker, Finite element methods for elliptic equations using nonconforming elements. Math. Comput. 31 (1977) 45-59.

[14] F. Bassi, L. Botti and A. Colombo, Agglomeration-based physical frame dG discretizations: An attempt to be mesh free. Math. Models Methods Appl. Sci. 24 (2014) 1495-1539.

[15] F. Bassi, L. Botti, A. Colombo, D.A. Di Pietro and P. Tesini, On the flexibility of agglomeration based physical space discontinuous Galerkin discretizations. J. Comput. Phys. 231 (2012) 45-65.

[16] F. Bassi, L. Botti, A. Colombo and S. Rebay, Agglomeration based discontinuous Galerkin discretization of the Euler and Navier-Stokes equations. Comput. Fluids 61 (2012) 77-85.

[17] S.C. Brenner and J. Zhao, Convergence of multigrid algorithms for interior penalty methods. Appl. Numer. Anal. Comput. Math. 2 (2005) 3-18.

[18] S.C. Brenner, J. Cui and L.-Y. Sung, Multigrid methods for the symmetric interior penalty method on graded meshes. Numer. Linear Algebra Appl. 16 (2009) 481-501.

[19] A Buffa, T.J.R. Hughes and G Sangalli, Analysis of a multiscale discontinuous Galerkin method for convection-diffusion problems. SIAM J. Numer. Anal. 44 (2006) 1420-1440. 
[20] A. Cangiani, J. Chapman, E.H. Georgoulis and M. Jensen, On the stability of continuous-discontinuous Galerkin methods for advection-diffusion-reaction problems. J. Sci. Comput. 57 (2013) 313-330.

[21] A. Cangiani, E.H. Georgoulis and P. Houston, $h p$-version discontinuous Galerkin methods on polygonal and polyhedral meshes. Math. Models Methods Appl. Sci. 24 (2014) 2009-2041.

[22] A. Chernov, Optimal convergence estimates for the trace of the polynomial $L^{2}$-projection operator on a simplex. Math. Comput. 81 (2012) 765-787.

[23] P.G. Ciarlet, The Finite Element Method for Elliptic Problems. Vol. 4 of Stud. Math. Appl. North-Holland Publishing Co., Amsterdam (1978).

[24] B. Cockburn, An Introduction to the Discontinuous Galerkin Method for Convection-Dominated Problems. In Advanced numerical approximation of nonlinear hyperbolic equations (Cetraro, 1997). Springer, Berlin (1998) 151-268.

[25] B. Cockburn, G.E. Karniadakis and C.-W. Shu., Eds., Discontinuous Galerkin Methods. Theory, Computation and Applications. Papers from the 1st International Symposium held in Newport, RI, May 24-26 1999. In Lect. Notes Comput. Sci. Eng. Springer-Verlag, Berlin (2000).

[26] B. Cockburn, B. Dong and J. Guzmán, Optimal convergence of the original DG method for the transport-reaction equation on special meshes. SIAM J. Numer. Anal. 46 (2008) 1250-1265.

[27] B. Cockburn, B. Dong, J. Guzmán and J. Qian, Optimal convergence of the original DG method on special meshes for variable transport velocity. SIAM J. Numer. Anal. 48 (2010) 133-146.

[28] D.A. Di Pietro and A. Ern, Mathematical Aspects of Discontinuous Galerkin Methods. Vol. 69 of Math. Appl. Springer, Heidelberg (2012).

[29] X. Feng and O.A. Karakashian, Two-level additive Schwarz methods for a discontinuous Galerkin approximation of second order elliptic problems. SIAM J. Numer. Anal., 39 (2001) 1343-1365.

[30] E.H. Georgoulis, Discontinuous Galerkin methods on shape-regular and anisotropic meshes. D. Phil. thesis, University of Oxford (2003).

[31] E.H. Georgoulis, Inverse-type estimates on $h p$-finite element spaces and applications. Math. Comput. 77 (2008) $201-219$.

[32] E.H. Georgoulis and A. Lasis, A note on the design of $h p$-version interior penalty discontinuous Galerkin finite element methods for degenerate problems. IMA J. Numer. Anal. 26 (2006) 381-390.

[33] S. Giani and P. Houston, $h p$-Adaptive composite discontinuous Galerkin methods for elliptic problems on complicated domains. Num. Meth. Partial Differ. Eqs. 30 (2014) 1342-1367.

[34] P. Houston, C. Schwab and E. Süli, Stabilized $h p$-finite element methods for first-order hyperbolic problems. SIAM J. Numer. Anal. 37 (2000) 1618-1643.

[35] P. Houston and E. Süli, Stabilised $h p$-finite element approximation of partial differential equations with nonnegative characteristic form. Computing 66 (2001) 99-119.

[36] P. Houston, C. Schwab and E. Süli, Discontinuous $h p$-finite element methods for advection-diffusion-reaction problems. SIAM J. Numer. Anal. 39 (2002) 2133-2163.

[37] C. Johnson and J. Pitkäranta, An analysis of the discontinuous Galerkin method for a scalar hyperbolic equation. Math. Comput. 46 (1986) 1-26.

[38] G. Karypis and V. Kumar, A fast and highly quality multilevel scheme for partitioning irregular graphs. SIAM J. Sci. Comput. 20 (1999) 359-392.

[39] C. Lasser and A. Toselli, An overlapping domain decomposition preconditioner for a class of discontinuous Galerkin approximations of advection-diffusion problems. Math. Comput. 72 (2003) 1215-1238.

[40] K. Lipnikov, D. Vassilev and I. Yotov, Discontinuous Galerkin and mimetic finite difference methods for coupled Stokes-Darcy flows on polygonal and polyhedral grids. Numer. Math. (2013) 1-40.

[41] R. Muñoz-Sola, Polynomial liftings on a tetrahedron and applications to the $h p$-version of the finite element method in three dimensions. SIAM J. Numer. Anal. 34 (1997) 282-314.

[42] J. Nitsche, Über ein Variationsprinzip zur Lösung von Dirichlet Problemen bei Verwendung von Teilräumen, die keinen Randbedingungen unterworfen sind. Abh. Math. Sem. Uni. Hamburg 36 (1971) 9-15.

[43] I. Perugia and D. Schötzau, An hp-analysis of the local discontinuous Galerkin method for diffusion problems. J. Sci. Comput. 17 (2002) 561-571.

[44] T.E. Peterson, A note on the convergence of the discontinuous Galerkin method for a scalar hyperbolic equation. SIAM J. Numer. Anal. 28 (1991) 133-140.

[45] W.H. Reed and T.R. Hill, Triangular mesh methods for the neutron transport equation. Technical Report LA-UR-73-479, Los Alamos Scientific Laboratory (1973).

[46] C. Schwab, $p$ - and $h p$-Finite Element Methods: Theory and Applications in Solid and Fluid Mechanics. Numerical Mathematics and Scientific Computation. Oxford University Press (1998).

[47] E.M. Stein, Singular Integrals and Differentiability Properties of Functions. Princeton, University Press, Princeton, N.J. (1970).

[48] C. Talischi, G.H. Paulino, A. Pereira and I.F.M. Menezes, Polymesher: A general-purpose mesh generator for polygonal elements written in Matlab. Struct. Multidisc. Optim. 45 (2012) 309-328,.

[49] R. Verfürth, On the constants in some inverse inequalities for finite element functions. Technical Report 257, University of Bochum (1999).

[50] D. Wirasaet, E.J. Kubatko, C.E. Michoski, S. Tanaka, J.J. Westerink and C. Dawson, Discontinuous Galerkin methods with nodal and hybrid modal/nodal triangular, quadrilateral, and polygonal elements for nonlinear shallow water flow. Comput. Methods Appl. Mech. Engrg. 270 (2014) 113-149. 